\title{
An overlapping Domain Decomposition preconditioning method for monolithic solution of shear bands
}

\author{
Luc Berger-Vergiat*, Haim Waisman* \\ Department of Civil Engineering \& Engineering Mechanics, Columbia University, \\ New York, NY 10027
}

\begin{abstract}
Metals subjected to high strain rate impact often exhibit a sudden and profound drop in the material's load bearing capability, a ductile failure phenomenon known as shear banding. Shear bands, characterized as material instabilities, are driven by shear heating and described as narrow regions that have sustained intense plastic deformation and high temperature rise. This coupled thermo-mechanical localization problem can be formulated as a nonlinear system with two balance equations, momentum and energy, and two constitutive laws for elasticity and plasticity.

In our formulation, mixed finite elements are used to discretize the equations in space and an implicit finite difference scheme is used to advance the system in time, where at every time step, a Newton type method is used to solve the system monolitically. To that end, a block Jacobian matrix is formed analytically using Gâteaux derivatives. The resulting Jacobian is sparse, nonsymmetric and its sparsity pattern and eigenvalue content vary with the different stages of shear bands formation, consequently posing a significant challenge to iterative solvers.

To address this issue, an overlapping Domain Decomposition preconditioner that takes into account the physics of shear bands and the domain in which it forms, is proposed. The key idea is to resolve the shear band domain accurately while only solving the remaining domain approximately, with selective updates when necessary. The preconditioner is formed on the basis of an additive Schwartz method that is applied to a Schur complement partition of the system.
\end{abstract}

The proposed preconditioner is implemented in parallel and tested on three dif-

\footnotetext{
*Corresponding author

Email address: luc. berger@columbia.edu (Luc Berger-Vergiat)
} 
ferent benchmark problems as compared against off-the-shelf solvers. We study the effect of $h$ - and k- refinement that are obtained from Isogeometric descritizations of the system, the overlapping strategy and its parallel performance. Excellent performance is demonstrated in serial and parallel on all benchmark examples.

Keywords: Shear bands, Preconditioner, Multiphysics, Schur complement, Domain Decomposition, GMRES, parallel scalability

\section{Introduction and problem statement}

Shearbanding is the localization of plastic strain into narrow bands that is typically observed in metals (and other materials) undergoing high strain rate loading. Shear bands, characterized in the literature as material instabilities [1], are driven by shear heating and result in the material's reduced stress bearing capacity and fracture. Accurate modeling of shear bands is very challenging due to the complicated multiphysics and difficult numerical modeling it requires. The problem is a highly nonlinear, coupled thermo-mechanical problem with localized crack-like domains that require specialized discretizations and solvers.

Experimentally derived material models for shear bands describe plastic flow as being dependent on temperature, strain rate, and a hardening parameter [2]. While several models are available, all are similar in that increasing temperature (due to plastic work) has a softening effect, causing plastic flow to occur more readily, while increases in strain rate and the hardening parameter have a hardening effect.

The experimental work of [3] shows that shear bands form in three stages. In Stage 1 the material deforms homogeneously and the temperature rises uniformly. Stage 2 marks the onset of shear band localization with a localized temperature rise leading to strain softening effect. Finally, in Stage 3, severe localization and rapid softening occurs, a phenomenon known as stress-collapse, which indicates a sudden and large drop in the material's load bearing capability. For more discussion on the physics of shear bands, the reader is referred to the reviews in $[4,5,6]$.

Shear bands can be modeled by a set of four nonlinear partial differential equations (PDEs) [2, 7, 8], two of which are balance equations: conservation of momentum and thermal energy, and two elastic and plastic constitutive equations. Under the assumption of small deformations, these four coupled equations, writ- 
ten in tensorial notation, are

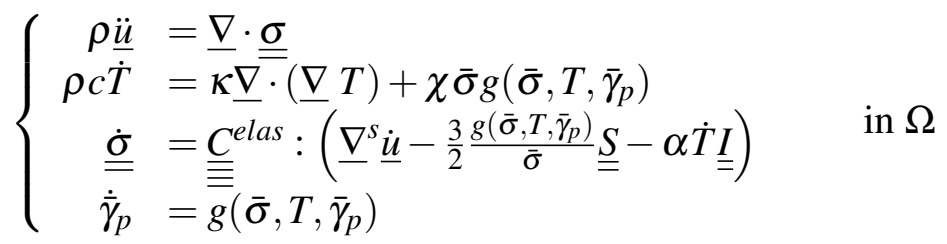

where one, two and four underlines indicate first-, second- and fourth- order tensors respectively; a colon (double dot) indicates the double contraction operator for tensors; an overline indicates an equivalent quantity; $\underline{\nabla} \bullet$ and $\underline{\nabla} \cdot \bullet$ are the gradient and the divergence operators and $\nabla^{S} \bullet$ is the symmetric part of the gradient operator, that is $\underline{\nabla}^{S} \bullet=\frac{1}{2}\left(\underline{\nabla} \bullet+(\underline{\nabla} \bullet)^{T}\right)$. The four unknown variables are the displacement field $\underline{u}$, the temperature field $T$, the stress field $\underline{\sigma}$ and the equivalent plastic strain (EQPS) field $\bar{\gamma}_{p} . \underline{\underline{S}}$ is the deviatoric part of $\underline{\underline{\sigma}}$ defined as $\underline{\underline{S}}=\underline{\underline{\sigma}}-\frac{\operatorname{tr}(\underline{\underline{\sigma}})}{3} \underline{\underline{I}}$ and $\bar{\sigma}$ is the Von Mises equivalent stress defined as $\bar{\sigma}=\sqrt{\frac{3}{2} \underline{\underline{S}}: \underline{\underline{S}}}$ (see [9]); $\underline{\underline{I}}$ is the second order identity tensor. The material parameters in the model are: $\rho$ the density, $c$ the specific heat, $\kappa$ the thermal conductivity, $\chi$ the Taylor-Quinney coefficient which defines the amount of plastic work converted to heat, $\alpha$ the thermal expansion coefficient, $\underline{\underline{\underline{E}}}^{\text {elas }}$ the elastic forth order tensor and the flow law $g$ that depends on the equivalent stress, the equivalent plastic strain and the temperature. An implicit expression for $g$ can be written by assuming that strain hardening, strain rate hardening and thermal softening effects are decomposed multiplicatively as follows

$$
\frac{\bar{\sigma}}{\sigma_{r e f}}=P(T) Q\left(\bar{\gamma}_{p}\right) R\left(\dot{\bar{\gamma}}_{p}\right)
$$

where $\sigma_{\text {ref }}$ is a reference stress flow. Rearranging the terms in (2) and assuming that $R$ is invertible, one can write

$$
g\left(\bar{\sigma}, T, \bar{\gamma}_{p}\right)=\dot{\bar{\gamma}}_{p}=R^{-1}\left(\frac{\bar{\sigma} / \sigma_{r e f}}{P(T) Q\left(\bar{\gamma}_{p}\right)}\right)
$$

$\Omega$ is the problem domain and its boundary $\Gamma=\Gamma_{d} \cup \Gamma_{n}$ and $\Gamma_{n} \cap \Gamma_{d}=\emptyset$, where $\Gamma_{d}$ and $\Gamma_{n}$ are the part of the boundary where Dirichlet and Neumann conditions are prescribed, respectively. The boundary conditions applied to the problem are as follows

$$
\begin{aligned}
& \underline{u}(\underline{x}, t)=\underline{\bar{u}}(\underline{x}, t) \quad \text { on } \Gamma_{d}^{u} \text {, } \\
& \underline{n} \cdot \underline{\sigma}(\underline{x}, t)=\bar{t}(\underline{x}, t) \text { on } \Gamma_{n}^{u} \text {, } \\
& T(\underline{x}, t)=\bar{T}(\underline{x}, t) \quad \text { on } \Gamma_{d}^{T} \text {, } \\
& \underline{n} \cdot \underline{q}(\underline{x}, t)=\bar{q}(\underline{x}, t) \quad \text { on } \Gamma_{n}^{T} \text {. }
\end{aligned}
$$


with the initial conditions stated as

$$
\begin{aligned}
& \underline{u}(\underline{x}, 0)=0 \\
& \underline{\bar{\sigma}}(\underline{x}, 0)=0 \\
& \bar{T}(\underline{x}, 0)=T_{0}(\underline{x}) \quad \text { in } \Omega \\
& \bar{\gamma}_{p}(\underline{x}, 0)=0
\end{aligned}
$$

where $T_{0}(\underline{x})$ is the initial temperature in the solid.

Multiple numerical schemes have been developed to analyze shear bands. The most common approach is to first simplify the governing equations in (1) by assuming adiabatic conditions in which $\kappa=0$. It is argued that since shear band propagation is a fast process and thermal diffusion is a slow one, the latter can be neglected in computations $[10,11,12]$. While such an approach simplifies the numerics significantly, it also results in a lack of a physical length scale in the system. Hence, in this case, the system is ill posed and the convergence will be sensitive to the mesh size $[13,14]$.

To this end, Liu et al. $[15,16]$ proposed a meshfree method and a multiresolution technique $[17,18]$ to regularize the adiabatic set of equations. However, this multiresolution method introduced three length scales: hardening scale, thermal diffusion scale and a micro-void spacing scale, which may not be trivial to determine. Other approaches such as viscoplasticity [19], gradient plasticity [20, 21, 22], thermal diffusion [23] and ductile damage models [24] have also been proposed to regularize the shear band problem.

If the adiabatic assumption is lifted and the thermal diffusion term is kept, then concurrent solution of the governing equations is required, which complicates the numerics. To keep the simulation at a low cost and to account for thermal diffusivity, splitting methods were introduced in [25, 26]. With these methods, the equations in system (1) are solved sequentially without the use of a global nonlinear solver. Nonetheless, when a shear band initiates (Stage 2 of the deformation state), numerical errors due to splitting methods cannot in general be recovered and have the tendency to compound through the simulation. Hence the numerical solution may diverge from experimental observations. More critically, these approaches do not always preserve the intrinsic length scale of the system and result in mesh dependent solutions. Other approaches using approximations of the Jacobian matrix, such as Picard iterations, have also similar properties as the splitting methods $[4,6]$.

To avoid splitting errors and mesh dependent results simultaneous solution methods, so called monolithic or Implicit Nonlinearly Consistent (INC) schemes 
are needed. The work by Batra et al. [4, 27, 23, 28, 29] and Waisman et al. $[30,31,32]$ show that concurrent solution of the equations leads to more reliable results that are insensitive to mesh size and mesh alignment. However this approach is more expensive as it requires the solution of a nonlinear set of equations at every time step. Moreover, due to the multiphysics nature of the problem, the discretized system is typically nonsymmetric and limits the choice of available solvers. In addition, one needs to compute the Jacobian of the nonlinear system in (1), which can be done numerically using a finite difference approach $[33,34,35,36]$ or analytically, thus reducing some of the numerical burden [30]. The matrix associated with the INC scheme is much larger than that used in splitting methods (combined with explicit schemes) but typically requires fewer time steps since the stability requirements are less restrictive [37]. Nonetheless, the bottleneck of such monolithic schemes is the efficiency of the linear solver which may not be suited for these thermo-mechanical systems.

In order to efficiently solve such linear systems, fast and robust preconditioners for Krylov-type iterative solvers, such as GMRES [38] or BICGStab [39], must be developed. The typical off-the-shelf algebraic preconditioners such as Jacobi, ILU or algebraic multigrid [40, 41] have difficulties accelerating the solution of Eq. 1 due to the highly nonlinear multiphysics nature of the problem, and the strong coupling of the equations.

Many preconditioners for multiphysics-type problems have been proposed. For example Ipsen [42] proposed a general method based on a Schur complement to precondition nonsymmetric matrices and his work has led to the widely used block Schur preconditioners for coupled physics problems [43]. Other block and non-block preconditioners have also been successfully applied to many physical problems, for instance fluids flow [44, 45, 46], solid mechanics [47, 48], magnetohydrodynamic [49] and mechanics [50, 51].

Another method to accelerate and/or parallelize the solution of multiphysics problems is a Domain Decomposition type approach. Common Domain Decomposition methods are those based on the Finite Element Tearing and Interconnecting (FETI) method $[52,53]$ which are iterative substructuring type methods, the overlapping Schwarz method [54, 55, 56, 57], or the variational multiscale enrichment method [58].

In solid mechanics problems involving plasticity, the Jacobian matrix varies greatly with the state of the system, and thus even if a preconditioner is effective at one time step, it may not be so at another. Therefore a reliable preconditioner must take advantage of the physics in order to offer significant speed up to the krylov solver. 
In this paper an overlapping Domain Decomposition type preconditioner to GMRES that takes advantage of the specific discretization and physics of the problem is proposed and investigated. The method is based on first, elimination of the stress and equivalent plastic strain fields using a Schur complement, and second, the resulting thermo-mechanical system is solved using a GMRES solver preconditioned with an additive Schwarz Domain Decomposition algorithm. The key idea is to decompose the computational domain into two subdomains: a so called 'healthy' subdomain where only mild plasticity is expected and a 'shear band' subdomain where significant plasticity and high temperature rise are dominant and lead to the localization of plastic strain. Such Domain Decomposition strategy will allow us to use expensive solvers in the shear band subdomain (where those are necessary) while an inexpensive solver in the healthy part. The solver is inexpensive in the sense that the approximation can be reused for many time steps throughout the analysis and only selectively updated as opposed to expensive updates at every time step. The proposed approach conceptually resembles the Domain Decomposition preconditioning method we proposed in $[59,60]$ although the previous work was applied to linear elastic fracture mechanics with the extended finite element method and no reuse of information was proposed then which is the focus of this work.

The proposed preconditioner is tested on three different benchmark problems and compared against off-the-shelf solvers. We study the effect of $h$ - and k- refinements that are obtained from Isogeometric descritizations of the system, the overlapping strategy and its parallel performance. Excellent performance is demonstrated in serial and parallel on all benchmark examples.

The paper is organized as follows. In section 2 we present the algebraic system of equations which arises from discretization of the governing PDE set in Eq. 1 and the implicit scheme used to solve and advance the system in time. In section 3 we derive the preconditioning algorithm and explain how its particular structure is well adapted to solve Eq. 1. In section 4 we analyze the performance of the proposed preconditioner on benchmark examples in serial and parallel. Final concluding remarks are provided in section 5.

\section{Discrete system and solution algorithm}

The governing equations in (1) are discretized using mixed element formulations, following the approach described in [30, 31]. We employ Isogeometric Analysis (IGA) [61, 62, 63] in which NURBS shape functions are used to disceretize the displacement and temperature fields while an irreducible description 
for the stress and equivalent plastic strain (EQPS) fields. In other words, the stress and equivalent plastic strain (EQPS) fields are sampled at Gauss points. Such irreducible-NURBS element was introduced in [32] for accurate solution of shear bands and is referred to as INSQ element.

In mixed finite element formulation of Eq. 1, the unknown variables vector $\mathbf{x}$ and the corresponding residual vector $\mathbf{R}$ are defined as follows

$$
\mathbf{x}=\left[\begin{array}{c}
\mathbf{x}_{u} \\
\mathbf{x}_{T} \\
\mathbf{x}_{\sigma} \\
\mathbf{x}_{\bar{\gamma}_{p}}
\end{array}\right] \quad \mathbf{R}=\left[\begin{array}{c}
\mathbf{R}_{u} \\
\mathbf{R}_{T} \\
\mathbf{R}_{\sigma} \\
\mathbf{R}_{\bar{\gamma}_{p}}
\end{array}\right]
$$

where the lower case letters indicate the corresponding fields.

An implicit Newmark time stepping scheme is used to advance the system in time together with a Newton-Raphson algorithm to solve the resulting nonlinear discrete equations at every time step. Let the nonlinear residual of the system at time step $i$ be written as $\mathbf{R}\left(\mathbf{x}^{i}\right)=0$, then the updated solution of the problem at the $k^{\text {th }}$ Newton iteration is $\mathbf{x}^{i, k+1}=\mathbf{x}^{i, k}+\delta \mathbf{x}^{i, k}$, where $\delta \mathbf{x}^{i, k}$ is the solution of the following equation

$$
\mathbf{J}\left(\mathbf{x}^{i, k}\right) \delta \mathbf{x}^{i, k}=-\mathbf{R}\left(\mathbf{x}^{i, k}\right) .
$$

It has been shown in [30] that a mixed finite element formulation leads to the following block structure of the Jacobian matrix

$$
\mathbf{J}=\left[\begin{array}{cccc}
\mathbf{J}_{u u} & \mathbf{0} & \mathbf{J}_{u \sigma} & \mathbf{0} \\
\mathbf{0} & \mathbf{J}_{T T} & \mathbf{J}_{T \sigma} & \mathbf{J}_{T \bar{\gamma}_{p}} \\
\mathbf{J}_{\sigma u} & \mathbf{J}_{\sigma T} & \mathbf{J}_{\sigma \sigma} & \mathbf{J}_{\sigma \bar{\gamma}_{p}} \\
\mathbf{0} & \mathbf{J}_{\bar{\gamma}_{p} T} & \mathbf{J}_{\bar{\gamma}_{p} \sigma} & \mathbf{J}_{\bar{\gamma}_{p} \bar{\gamma}_{p}}
\end{array}\right]
$$

where the first subscript of each block indicates which residual equation was differentiated and the second subscript indicates the variable it is being differentiated with respect to (e.g. $\mathbf{J}_{u \sigma}=\frac{\partial \mathbf{R}_{u}}{\partial \sigma}$ ). After discretization, the block structure can be further expanded as follow

$$
\mathbf{J}=\left[\begin{array}{cccc}
\mathbf{M}_{u} & \mathbf{0} & \mathbf{K}_{u} & \mathbf{0} \\
\mathbf{0} & \mathbf{M}_{T}+\mathbf{K}_{T}+\mathbf{G}_{T T} & \mathbf{G}_{T \sigma} & \mathbf{G}_{T \bar{\gamma}_{p}} \\
\mathbf{K}_{\sigma} & \mathbf{M}_{\sigma T}+\mathbf{G}_{\sigma T} & \mathbf{M}_{\sigma}+\mathbf{G}_{\sigma \sigma} & \mathbf{G}_{\sigma \bar{\gamma}_{p}} \\
\mathbf{0} & \mathbf{G}_{\bar{\gamma}_{p} T} & \mathbf{G}_{\bar{\gamma}_{p} \sigma} & \mathbf{M}_{\bar{\gamma}_{p}}+\mathbf{G}_{\bar{\gamma}_{p} \bar{\gamma}_{p}}
\end{array}\right]
$$

where $\mathbf{M}$ denotes a mass matrix, $\mathbf{K}$ a stiffness matrix arising from linear material behavior and $\mathbf{G}$ is a matrix corresponding to the linearization of the flow law in 
Eq. 2. Note that the Jacobian matrix is non symmetric which limits the choice of available linear solvers. Furthermore, when the solid is in the linear elastic deformation regime, all $\mathbf{G}$ blocks vanish and the structure of $\mathbf{J}$ is simplified to

$$
\mathbf{J}=\mathbf{J}^{l i n}=\left[\begin{array}{cccc}
\mathbf{J}_{u u} & \mathbf{0} & \mathbf{J}_{u \sigma} & \mathbf{0} \\
\mathbf{0} & \mathbf{J}_{T T} & \mathbf{0} & \mathbf{0} \\
\mathbf{J}_{\sigma u} & \mathbf{J}_{\sigma T} & \mathbf{J}_{\sigma \sigma} & \mathbf{0} \\
\mathbf{0} & \mathbf{0} & \mathbf{0} & \mathbf{J}_{\bar{\gamma}_{p} \bar{\gamma}_{p}}
\end{array}\right]=\left[\begin{array}{cccc}
\mathbf{M}_{u} & \mathbf{0} & \mathbf{K}_{u} & \mathbf{0} \\
\mathbf{0} & \mathbf{M}_{T}+\mathbf{K}_{T} & \mathbf{0} & \mathbf{0} \\
\mathbf{K}_{\sigma} & \mathbf{M}_{\sigma T} & \mathbf{M}_{\sigma} & \mathbf{0} \\
\mathbf{0} & \mathbf{0} & \mathbf{0} & \mathbf{M}_{\bar{\gamma}_{p}}
\end{array}\right] .
$$

Up to this point all the properties of the Jacobian matrix described in equations (8)-(10) are intrinsic to the mechanical system and completely independent of the discretization used.

Next, we discuss the special properties of INSQ elements that are important for development of the preconditioner in section 3. First, note that the irreducible discretization used for the stress and EQPS fields are spatially decoupled which

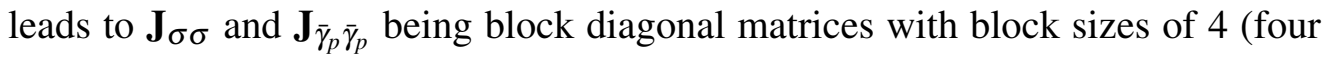
stresses) and 1 (one equivalent plastic strain), respectively. Furthermore in the linear elastic regime $\mathbf{J}_{\sigma \sigma}$ is diagonal since the derivatives of the flow law with respect to the variables of the problem are zero. In addition the coupled stress-plasticity term $\mathbf{J}_{\bar{\gamma}_{p} \sigma}$ and the temperature-plasticity term $\mathbf{J}_{\bar{\gamma}_{p} T}$ both vanish. However, note that $\mathbf{J}_{\bar{\gamma}_{p}} \bar{\gamma}_{p}$ remains a diagonal matrix due to the mass matrix term, as shown in Eq. 10. Also note that the temperature-stress term is only one way coupled and hence $\mathbf{J}_{T \sigma}$ vanishes too. Hence, the sparsity pattern of the Jacobian matrix is evolving with the deformation state as illustrated in Fig. 1 for the linear elastic and plastic regimes.

In irreducible formulations, it is more convenient for notational purposes to re-group the irreducible blocks (stress and plastic strain) under one augmented matrix $\mathbf{J}_{\eta \eta}$ as follows

$$
\mathbf{J}_{\eta \eta}=\left[\begin{array}{ll}
\mathbf{J}_{\sigma \sigma} & \mathbf{J}_{\sigma \bar{\gamma}_{p}} \\
\mathbf{J}_{\bar{\gamma}_{p} \sigma} & \mathbf{J}_{\bar{\gamma}_{p} \bar{\gamma}_{p}}
\end{array}\right]
$$

and to re-group the equilibrium blocks (displacement and temperature) under $\mathbf{J}_{\alpha \alpha}$ as follows

$$
\mathbf{J}_{\alpha \alpha}=\left[\begin{array}{ll}
\mathbf{J}_{u u} & \mathbf{J}_{u T} \\
\mathbf{J}_{T u} & \mathbf{J}_{T T}
\end{array}\right]
$$

Plugging these expressions into Eq. 8 leads to the following more condensed expression for the Jacobian matrix

$$
\mathbf{J}=\left[\begin{array}{ll}
\mathbf{J}_{\alpha \alpha} & \mathbf{J}_{\alpha \eta} \\
\mathbf{J}_{\eta \alpha} & \mathbf{J}_{\eta \eta}
\end{array}\right]
$$



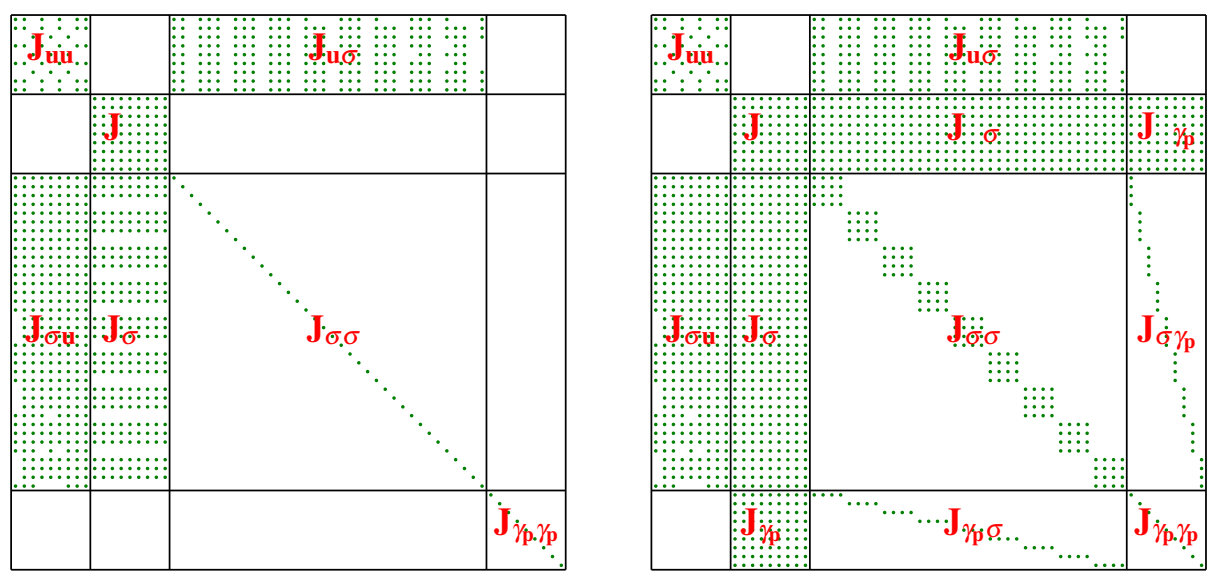

Figure 1: Sparsity pattern of a single Jacobian element matrix in the linear elastic (left) and nonlinear plastic (right) regime. The green dots indicate nonzero terms and the black lines show the limits of the blocks in the matrix.

and the system of linear equations that needs to be solved becomes

$$
\left[\begin{array}{ll}
\mathbf{J}_{\alpha \alpha} & \mathbf{J}_{\alpha \eta} \\
\mathbf{J}_{\eta \alpha} & \mathbf{J}_{\eta \eta}
\end{array}\right]\left[\begin{array}{l}
\mathbf{x}_{\alpha} \\
\mathbf{x}_{\eta}
\end{array}\right]=-\left[\begin{array}{l}
\mathbf{R}_{\alpha} \\
\mathbf{R}_{\eta}
\end{array}\right]
$$

where the $\alpha$ and $\eta$ variables can be used in the more efficient blocked format, as shown in Fig. 2.

\section{Proposed Domain Decomposition preconditioner for the linearized system}

The proposed solver is constructed in three steps. First, a Schur complement is applied to the system in Eq. 14 and the primary fields are reduced to displacements and temperature. Second, the Schur system is decomposed using a Domain Decomposition method, by defining a shear band subdomain and a so called healthy subdomain. The shearband subdomain consists of a region where intense shearing, severe plastic localization and significant heating is expected, while the healthy subdomain corresponds to a more or less homogeneous deformation region with non significant temperature rise. Third, to take advantage of these two subdomains, the shear band domain is accurately resolved by an LU direct solve at every step while the healthy domain is approximated selectively. In other words, 

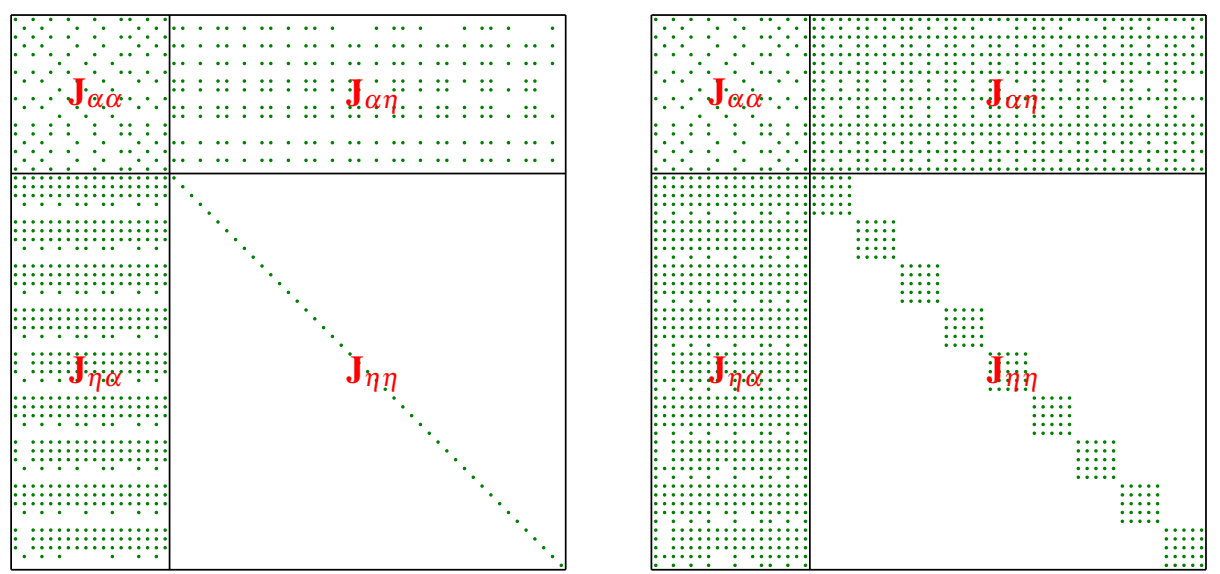

Figure 2: Sparsity pattern of a single Jacobian element matrix in the linear elastic (left) and nonlinear plastic (right) regime, with nested $\alpha$ and $\eta$ variables. The green dots indicate nonzero terms and the black lines show the limits of the blocks in the matrix.

the approximated solution in the healthy domain is carried out throughout the analysis and only when a certain threshold of maximum iterations is reached, updated. The following subsection detail the three steps used to construct the preconditioner.

\subsection{Step I: field-split Schur complement approach}

We start with a brief introduction to the Schur complement method [64]. Consider the following linear system

$$
\mathbf{J} \delta \mathbf{x}=\mathbf{R}
$$

Using a partition of the unknowns $\delta \mathbf{x}=\left[\begin{array}{l}\delta \mathbf{x}_{0} \\ \delta \mathbf{x}_{1}\end{array}\right]$ and residuals $\mathbf{R}=\left[\begin{array}{l}\mathbf{R}_{0} \\ \mathbf{R}_{1}\end{array}\right]$, it is convenient to rewrite Eq. 15 in the following form

$$
\left[\begin{array}{ll}
\mathbf{J}_{00} & \mathbf{J}_{01} \\
\mathbf{J}_{10} & \mathbf{J}_{11}
\end{array}\right]\left[\begin{array}{l}
\delta \mathbf{x}_{0} \\
\delta \mathbf{x}_{1}
\end{array}\right]=\left[\begin{array}{l}
\mathbf{R}_{0} \\
\mathbf{R}_{1}
\end{array}\right]
$$

Furthermore, assuming that the $\mathbf{J}_{11}$ block matrix in the partitioned system of Eq. 16 is invertible, one can rewrite $\mathbf{J}$ as the product of three matrices, two of 
which are triangular with identity blocks on their diagonal and a block diagonal matrix, as follows

$$
\left[\begin{array}{ll}
\mathbf{J}_{00} & \mathbf{J}_{01} \\
\mathbf{J}_{10} & \mathbf{J}_{11}
\end{array}\right]=\left[\begin{array}{cc}
\mathbf{1} & \mathbf{J}_{01} \mathbf{J}_{11}^{-1} \\
\mathbf{0} & \mathbf{1}
\end{array}\right]\left[\begin{array}{cc}
\mathbf{S} & \mathbf{0} \\
\mathbf{0} & \mathbf{J}_{11}
\end{array}\right]\left[\begin{array}{cc}
\mathbf{1} & \mathbf{0} \\
\mathbf{J}_{11}^{-1} \mathbf{J}_{10} & \mathbf{1}
\end{array}\right]
$$

where

$$
\mathbf{S}=\mathbf{J}_{00}-\mathbf{J}_{01} \mathbf{J}_{11}^{-1} \mathbf{J}_{10}
$$

is the Schur complement of block $\mathbf{J}_{11}$ in Eq. 17. The alternate expression of $\mathbf{J}$ given in Eq. 17 is particularly useful since one can compute its inverse based on the inverses of $\mathbf{J}_{11}$ and $\mathbf{S}$ :

$$
\begin{aligned}
\mathbf{J}^{-1} & =\left[\begin{array}{cc}
\mathbf{1} & \mathbf{0} \\
-\mathbf{J}_{11}^{-1} \mathbf{J}_{10} & \mathbf{1}
\end{array}\right]\left[\begin{array}{cc}
\mathbf{S}^{-1} & \mathbf{0} \\
\mathbf{0} & \mathbf{J}_{11}^{-1}
\end{array}\right]\left[\begin{array}{cc}
\mathbf{1} & -\mathbf{J}_{01} \mathbf{J}_{11}^{-1} \\
\mathbf{0} & \mathbf{1}
\end{array}\right] \\
& =\left[\begin{array}{cc}
\mathbf{S}^{-1} & -\mathbf{S}^{-1} \mathbf{J}_{01} \mathbf{J}_{11}^{-1} \\
-\mathbf{J}_{11}^{-1} \mathbf{J}_{10} \mathbf{S}^{-1} & \mathbf{J}_{11}^{-1}+\mathbf{J}_{11}^{-1} \mathbf{J}_{10} \mathbf{S}^{-1} \mathbf{J}_{01} \mathbf{J}_{11}^{-1}
\end{array}\right]
\end{aligned}
$$

This expression of the inverse of $\mathbf{J}$ shows that if $\mathbf{J}_{11}$ can be easily inverted then most of the computational effort should be concentrated on computing the inverse of $\mathbf{S}$.

Remark 1: In practice $\mathbf{J}^{-1}$ is never computed using Eq. 19 since it is too expensive to form. Instead a gaussian elimination applied to the left hand side of Eq. 16 is done using the expression of $\mathbf{J}$ in Eq. 17. This method allows to solve the system using matrix-vector multiplications rather than matrix-matrix multiplications.

Coming back to our specific shearband system, in order to reduce the cost of inverting $\mathbf{J}$, a field-split into constitutive/conservation laws is proposed. In other words, we partition the variables in two groups, $\alpha$ and $\eta$, corresponding to (displacement, temperatures) and (stress, equivalent plastic strain), respectively. Note that the $\eta$ variables for the irreducible family of elements, used in this paper, correspond to the variables which are localized at Gauss points (history variables). Hence, inverting $\mathbf{J}_{\eta \eta}$ can be done efficiently at the element level. This allows us 
to expand the Schur complement defined in Eq. 17 as indicated below

$$
\begin{aligned}
\mathbf{S} & =\left[\begin{array}{ll}
\mathbf{S}_{u u} & \mathbf{S}_{u T} \\
\mathbf{S}_{T u} & \mathbf{S}_{T T}
\end{array}\right] \\
& =\mathbf{J}_{\alpha \alpha}-\mathbf{J}_{\alpha \eta} \mathbf{J}_{\eta \eta}^{-1} \mathbf{J}_{\eta \alpha} \\
& =\left[\begin{array}{cc}
\mathbf{J}_{u u}-\mathbf{J}_{u \eta} \mathbf{J}_{\eta \eta}^{-1} \mathbf{J}_{\eta u} & -\mathbf{J}_{u \eta} \mathbf{J}_{\eta \eta}^{-1} \mathbf{J}_{\eta T} \\
-\mathbf{J}_{T \eta} \mathbf{J}_{\eta \eta}^{-1} \mathbf{J}_{\eta u} & \mathbf{J}_{T T}-\mathbf{J}_{T \eta} \mathbf{J}_{\eta \eta}^{-1} \mathbf{J}_{\eta T}
\end{array}\right]
\end{aligned}
$$

and Eq. 7 is then solved as follows

$$
\left\{\begin{aligned}
\mathbf{S} \delta \mathbf{x}_{\alpha} & =\mathbf{R}_{\alpha}-\mathbf{J}_{\alpha \eta} \mathbf{J}_{\eta \eta}^{-1} \mathbf{R}_{\eta} \\
\delta \mathbf{x}_{\eta} & =\mathbf{J}_{\eta \eta}^{-1}\left(\mathbf{R}_{\eta}-\mathbf{J}_{\eta \alpha} \delta \mathbf{x}_{\alpha}\right)
\end{aligned}\right.
$$

The first equation in system (21) requires the solution of a linear system of equations whereas the second equation only requires matrix vector multiplications. In the rest of this section we will focus on the approach used to solve Eq. 21a using a preconditioned GMRES solver.

\subsection{Step II: Domain Decomposition of the Schur complement system}

Since most of the significant deformation, plasticity and heating takes place in the narrow shear band region while only minor plasticity and heating happen out of this region, a preconditioner that takes advantage of this physics would be quite efficient. Hence, a preconditioner that decomposes the domain and concentrate more effort in the shear band domain while reusing information away from the band could lead to a significant improvement in computational performance. This leads us to propose a Domain Decomposition approach to precondition the GMRES solver used in Eq. 21a, as represented in Fig. 3.

Such Domain Decomposition approach allows us to use different operators in the shear band subdomain $\Omega_{s b}$, and the healthy subdomain $\Omega_{h}$ in order to solve the Schur complement system efficiently. Moreover, an overlapping scheme may be associated with each subdomain and defined by a length parameter, $\delta_{h}$ and $\delta_{s b}$ for the healthy and shear band subdomains, respectively. For example consider the impact problem shown in Fig. $3 \mathrm{a}$ and the corresponding equivalent plastic strain that localizes in the plate at the end of the simulation (Stage 3, stress collapse state), as shown in Fig. 3b. As mentioned above, two subdomains are formed $\Omega_{s b}$, shown in Fig. 3c and $\Omega_{h}$, shown in Fig. 3d note that width of the subdomains 
presented in these figures are increased for the purpose of illustration but in the actual simulations are smaller. Each of those subdomains may have overlapping regions as shown in these figures, denoted by $\delta_{s b}$ for the shear band subdomain in Fig. $3 \mathrm{c}$ and healthy subdomain $\delta_{h}$ in Fig. $3 \mathrm{~d}$.

In the current work, an additive Schwarz type domain decomposition method is proposed. To this end, we define a boolean restriction operator $\mathbf{R}_{i}^{\delta}$ as a square matrix with diagonal entries set to 1 , if the corresponding degree of freedom belongs to a node in subdomain $i$ or within the overlap region $\delta_{i}$, otherwise it is set to 0 . All non diagonal entries are zeros. That is

$$
\mathbf{R}_{i}^{\delta_{i}}=\left[\begin{array}{cccccc}
1 & 0 & \ldots & & & \\
0 & 1 & 0 & \ldots & & \\
& 0 & 0 & 0 & \ldots & \\
& & 0 & \ddots & 0 & \ldots
\end{array}\right] .
$$

With this expression for the restriction operator, one can define the restriction of the Schur complement matrix into the two subdomain as

$$
\mathbf{S}_{h}=\mathbf{R}_{h}^{\delta_{h}} \mathbf{S R}_{h}^{\delta_{h}} \quad \mathbf{S}_{s b}=\mathbf{R}_{s b}^{\delta_{s b}} \mathbf{S R}_{s b}^{\delta_{s b}} .
$$

Note that such definition is general and allows for the same nodes to be defined in the both domain.

Remark 2: Note that even though $\mathbf{S}_{i}$ are not invertible due to the expression of $\mathbf{R}_{i}^{\delta_{i}}$, one can invert their restriction to the subspace of vectors spanned by $\Omega_{i}$ in $\mathbb{R}^{n}$. Therefore we adopt the following notation

$$
\mathbf{S}_{h}^{-1} \equiv\left(\left(\mathbf{S}_{h}\right)_{\mid \Omega_{h}}\right)^{-1} \quad \mathbf{S}_{s b}^{-1} \equiv\left(\left(\mathbf{S}_{s b}\right)_{\mid \Omega_{s b}}\right)^{-1} .
$$

Note that while the overlap region is not explicitly written in Eq. 24 it is implicitly included in the restriction of $\Omega$ to $\Omega_{i}$.

A matrix form of of the proposed Domain Decomposition preconditioner can be written as follows

$$
\mathbf{P}_{A S M}^{-1}=\mathbf{S}_{h}^{-1}+\mathbf{S}_{s b}^{-1}
$$

Note that the subscript ASM refers to the additive Schwarz method and the flexibility of such formulation will allow for different solvers in the two different subdomains.

More details on the additive Schwarz method can be found in [54, 65] and on the restricted additive Schwarz variant, that is employed in this work, in [50, 


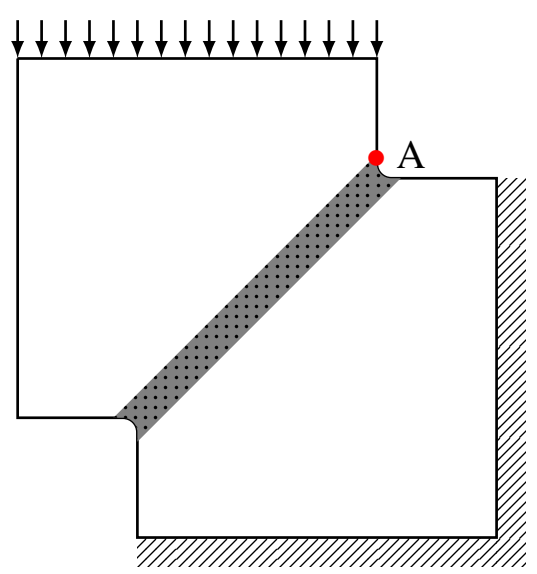

(a) High strain rate impact onto a steel plate

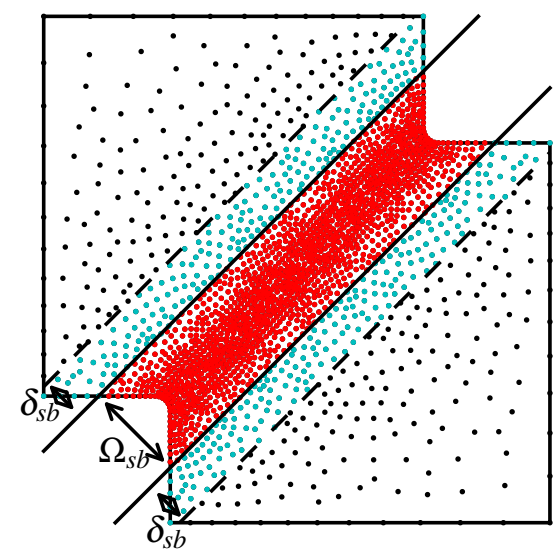

(c) The shear band subdomain $\Omega_{s b}$ (marked by red nodes $\bullet$ ) and its overlapping region $\delta_{s b}$ (marked in cyan •)

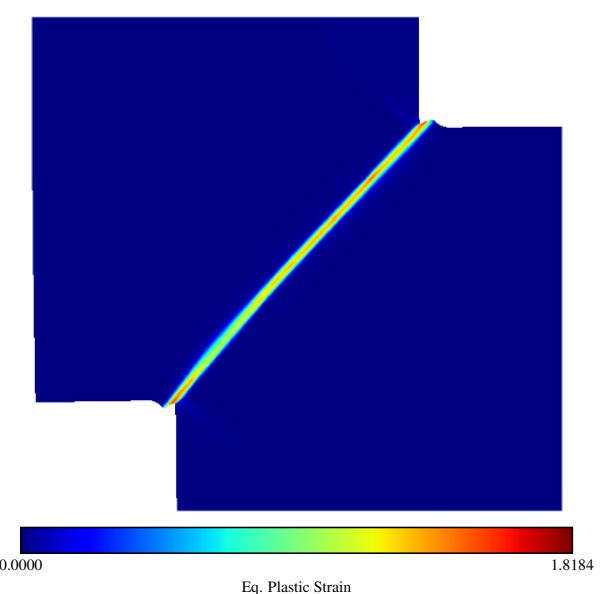

(b) Localization of the equivalent plastic strain field at the end of the simulation

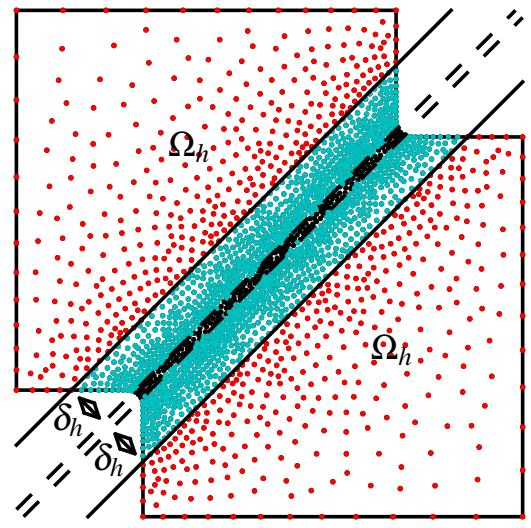

(d) The healthy domain $\Omega_{h}$ (marked by red nodes $\bullet$ ) and its overlapping region $\delta_{h}$ (marked in cyan •)

Figure 3: An overlapping Domain Decomposition approach for the shear band problem shown in Fig. 3a. The dots in the figure denote the FE nodes. Note that two different solvers are proposed for the two subdomains. 
66]. Additionally, more detailed derivation of the additive Schwarz method is also provided in Appendix A.

With these notations, the preconditioned form of Eq. 21a to be solved iteratively with a GMRES algorithm is

$$
\mathbf{P}_{A S M}^{-1} \mathbf{S x}_{\alpha}=\mathbf{P}_{A S M}^{-1}\left(\mathbf{R}_{\alpha}-\mathbf{J}_{\alpha \eta} \mathbf{J}_{\eta \eta}^{-1} \mathbf{R}_{\eta}\right)
$$

plugging the definition in Eq. 25 we get

$$
\left(\mathbf{S}_{h}^{-1}+\mathbf{S}_{s b}^{-1}\right) \mathbf{S x}_{\alpha}=\left(\mathbf{S}_{h}^{-1}+\mathbf{S}_{s b}^{-1}\right)\left(\mathbf{R}_{\alpha}-\mathbf{J}_{\alpha \eta} \mathbf{J}_{\eta \eta}^{-1} \mathbf{R}_{\eta}\right)
$$

Hence, two different operators can be used for $\mathbf{S}_{h}$ and $\mathbf{S}_{s b}$ in order to accelerate the convergence of the linear solver, which is the objective of this paper.

\subsection{Step III: Subdomains solution strategy}

To lower the cost of computing $\mathbf{P}_{A S M}^{-1}$ the key idea is to compute the inverse of $S_{h}$ at the first Newton iteration of the first time step (a linear elastic step with the operator $\left(\mathbf{S}_{h}^{l i n}\right)^{-1}$ ) and then reuse the same approximated solution throughout the analysis. Nonetheless, the operator can also be adaptively updated if a certain threshold on the maximum allowable number of linear iterations is reached.

Such an approach leads to significant CPU savings since the healthy subdomain does not sustain significant amount of plasticity or heating and remains homogeneous (i.e. no localization happens in this domain). Hence, the Schur complement operator of the healthy subdomain may be written as

$$
\begin{aligned}
\mathbf{S}_{h}^{l i n} & =\left[\begin{array}{cc}
\mathbf{M}_{u} & \mathbf{0} \\
\mathbf{0} & \mathbf{M}_{T}+\mathbf{K}_{T}
\end{array}\right]-\left[\begin{array}{cc}
\mathbf{K}_{u} & \mathbf{0} \\
\mathbf{0} & \mathbf{0}
\end{array}\right]\left[\begin{array}{cc}
\mathbf{M}_{\sigma} & \mathbf{0} \\
\mathbf{0} & \mathbf{M}_{\bar{\gamma}_{p}}
\end{array}\right]^{-1}\left[\begin{array}{cc}
\mathbf{K}_{\sigma} & \mathbf{M}_{\sigma T} \\
\mathbf{0} & \mathbf{0}
\end{array}\right] \\
& =\left[\begin{array}{cc}
\mathbf{M}_{u}-\mathbf{K}_{u} \mathbf{M}_{\sigma}^{-1} \mathbf{K}_{\sigma} & -\mathbf{K}_{u} \mathbf{M}_{\sigma}^{-1} \mathbf{M}_{\sigma T} \\
\mathbf{0} & \mathbf{M}_{T}+\mathbf{K}_{T}
\end{array}\right]
\end{aligned}
$$

Remark 3: The off diagonal term in $\mathbf{S}_{h}^{\text {lin }}$ is due to the thermal expansion of the material. This term can easily be dropped since in the elastic regime no heat is produced and the heat diffused away from the shear band largely remains with in the shear band domain $\Omega_{s b}$, hence no significant thermal expansion occurs. The remaining diagonal terms are the elastic stiffness matrix and the linear thermal conductivity matrix. 
For certain problems, the $\mathrm{ASM}_{u}$ preconditioner may gradually become less effective as some plasticity can also develop in the healthy domain. To determine when an update is necessary we record the maximum number of GMRES iterations, denoted $n_{r e f}$ during the first time step. At that time, $\mathbf{S}_{h}$ and $\mathbf{S}_{h}^{\text {lin }}$ are almost identical assuming that little or no plasticity occurs during the first time step, thus the $\mathrm{ASM}_{u}$ preonditioner requires the same amount of iterations as the ASM preconditioner to converge. We can therefore set a parameter $\lambda$ so that an update of $\mathbf{S}_{h}^{\text {lin }}$ is triggered when

$$
n_{\text {cur }} \geq \lambda n_{\text {ref }}
$$

where $n_{c u r}$ is the maximum number of iterations required to obtain convergence at the current time step. At the time step when $\mathbf{S}_{h}^{l i n}$ is updated we also update the value of $n_{r e f}$. In this work, $\lambda$ is set to $\lambda=1.5$, which based on our experience provides good computational performance, however other values and different update methods could also be explored.

Reformulating the additive Schwarz preconditioner in Eq. 25 accordingly, we have

$$
\mathbf{P}_{A S M_{u}}^{-1}=\left(\mathbf{S}_{h}^{u}\right)^{-1}+\mathbf{S}_{s b}^{-1}
$$

where $\mathrm{ASM}_{u}$ stands for the additive Schwarz method with selective updates to the preconditioner and hence only a few solve are required to form $\mathbf{S}_{h}^{u}$. Furthermore, if no updates are done, the preconditioner reduces to its cheapest form

$$
\mathbf{P}_{A S M_{u}}^{-1}=\left(\mathbf{S}_{h}^{l i n}\right)^{-1}+\mathbf{S}_{s b}^{-1} .
$$

\subsection{Preconditioner implementation and parallelization}

In this section an overview of the implementation and packages used for parallel execution is presented. We omit some critical but independent aspect of the algorithm such as mesh generation, I/O, time integration and the Newton solver. Assuming that a mesh obtained from discretization of the problem is available, and that the healthy and shear band subdomains have been geometrically defined, we employ the FEM software (FEAP [67]) in our implementation with the package (METIS [68]) to obtain a partitioning of the mesh. Note that the partition of the mesh and the definition of the subdomains are two independent issues. The FEM software assigns a partition to each of the processor using an MPI library (MPICH [69]). In other words one MPI rank is assigned to each partition.

A pseudo algorithm for the solution of the system in Eq. 21 locally owned by each MPI rank is presented in Fig. 4. First, a Schur complement of Eq. 20 is formed taking advantage of the fact that stresses and equivalent plastic strain in 


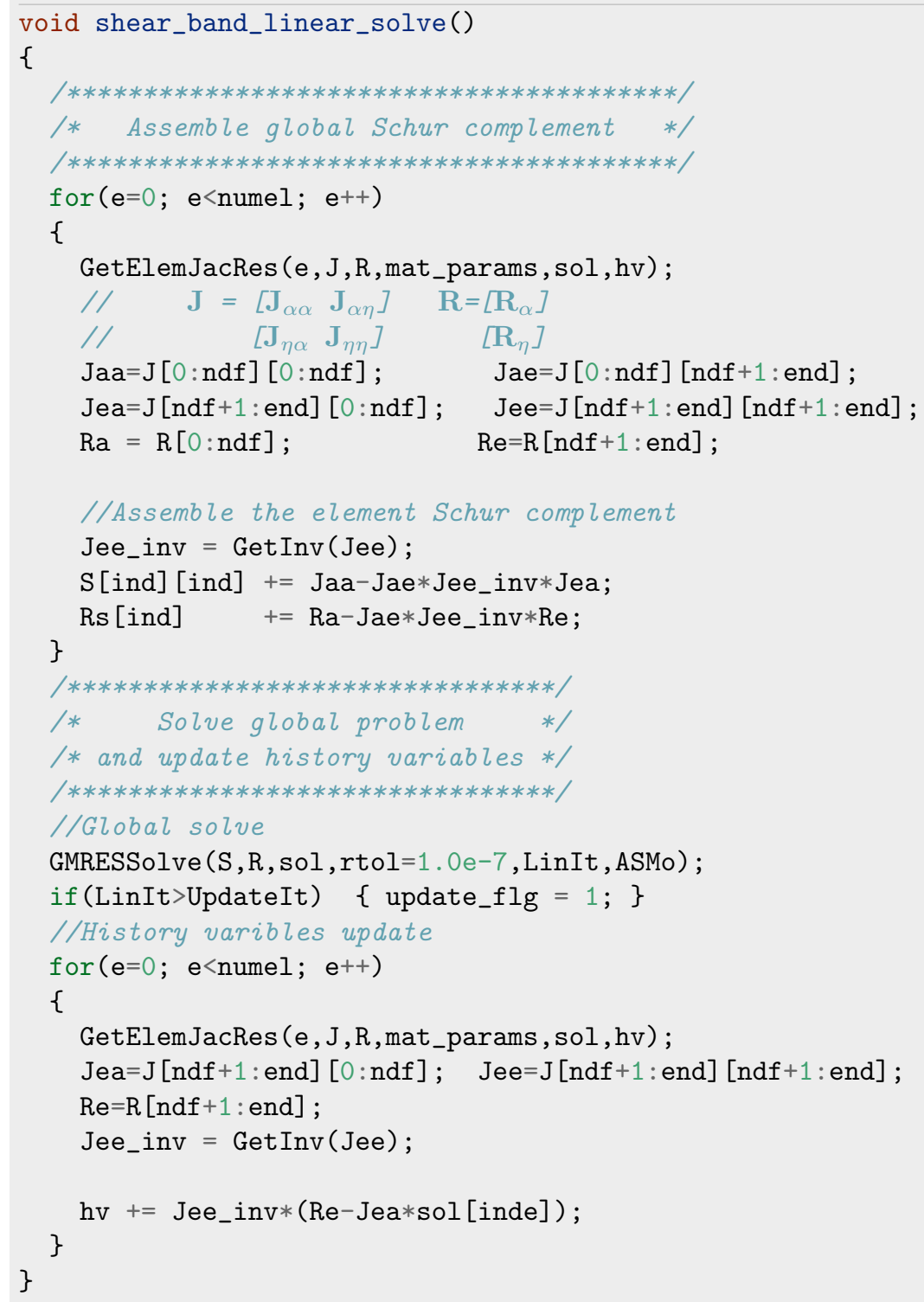

Figure 4: Pseudo algorithm for the solution of shear bands given in Eq. 1, note the call to the $\mathrm{ASM}_{u}$ preconditioner in global solve line. 
the INSQ element are state variables which are sampled at Gauss points. Hence, in our implementation this operation is done at the element level before assembly. Furthermore since the Gauss points within one element are decoupled, as can be seen in Fig. 2, the calculation of $\mathbf{J}_{\alpha \eta} \mathbf{J}_{\eta \eta}^{-1} \mathbf{J}_{\eta \alpha}$ can be optimized on a each rank with threading (OMP) and vectorized instructions (SSE,AVX), depending on the computer architecture.

Next, Eq. 31 is approximated with a GMRES solver predonditioned by $\mathbf{P}_{A S M_{u}}$, see the global solve line in Fig. 4, where the matrices $\mathbf{S}_{h}^{l i n}, \mathbf{S}_{h}^{u}$ and $\mathbf{S}_{s b}$ are as follows:

- $\mathbf{S}_{h}^{l i n}$ is factored at the first time step, the factors are stored and reused at each linear iteration during the simulation until an update to the preconditioner is made, as summarized in Section 3.3,

- $\mathbf{S}_{h}^{u}$ is a new update of the preconditioner which is factored, stored and reused in place of $\mathbf{S}_{h}^{l i n}$ when the maximum number of allowed linear iteration is reached.

- $\mathbf{S}_{s b}$ is inverted at each Newton iteration. The cost associated with the factorization of this matrix is reasonably low considering that the shear band is highly localized.

A pseudo algorithm for forming the preconditioners is summarized in Fig. 5, where MUMPS corresponds to the software library used to perform matrix decompositions.

\section{Numerical results}

In this section we present the convergence of the proposed Domain Decomposition preconditioner (denoted by $\mathrm{ASM}_{u}$ ) in terms of linear iterations and wallclock time. Four numerical studies are presented:

I. convergence comparison with state-of-practice solver/preconditioner pairs considering different mesh refinements

II. a study of the influence of overlap regions in an overlapping Domain Decomposition approach as controlled by the length parameter $\delta$

III. convergence study in terms of $h$ - and $k$-refinement when applied to a NURBS based element discretization

IV. parallel performance of the preconditioner to assess its scalability on multiple processors. 


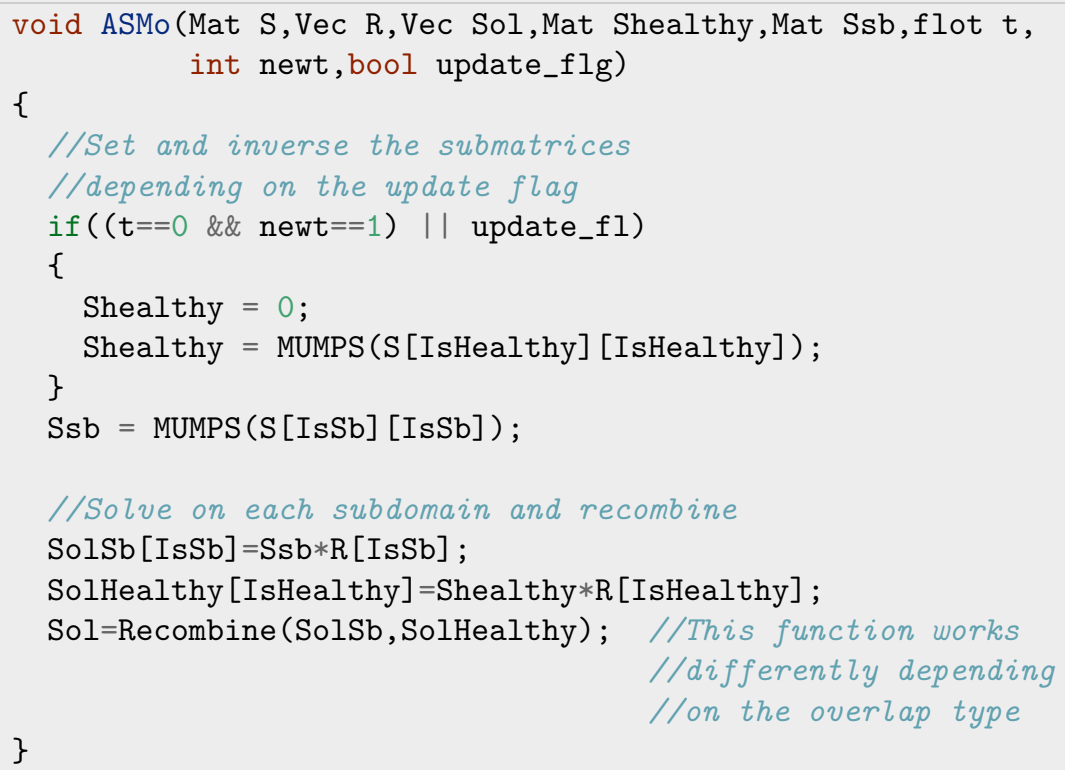

Figure 5: Pseudo algorithm for forming both subdomain preconditioners and updating the healthy subdomain matrix

The solvers used for the comparison are (i) PETSc's LU solver applied to $\mathbf{J}$ in Eq. 7 (denoted by LU(J)), (ii) PETSc's LU solver applied to $\mathbf{S}$ in Eq. 21a and (denoted by LU(S), (iii) a GMRES solver preconditioned with an ILU(0) algorithm applied to $\mathbf{S}$ in Eq. 21a (denoted by GMRES(S,ILU(0))).

LU direct solvers are the fastest to solve small sized systems and hence constitute a good comparison with the proposed Domain Decomposition solvers. The GMRES-ILU(0) solver is also a standard off-the-shelf, robust method that is widely used in scientific computing applications. Additionally, the proposed preconditioner $\mathrm{ASM}_{u}$ is also compared with another variant of this method, denoted by ASM, that uses $\mathbf{S}_{h}$ instead of $\mathbf{S}_{h}^{u}$ in the preconditioner. In other words, this option forms and solves the two subdomains at every time step and doesn't exploit the reuse idea. However, this is very useful since one can evaluate the appropriateness of the proposed reuse strategy outside of the shear band zone.

The numerical examples are run on a DELL optiplex 755 desktop with 4 cores at $2.83 \mathrm{GHz}$ and $4 \mathrm{~GB}$ of RAM. The PDE model is implemented using the Finite Element Analysis Program (FEAP) [67] and the iterative solver is implemented 
in PETSc [70, 71, 72], a relative residual tolerance of $10^{-7}$ is set as the threshold for convergence. MUMPS [73, 74, 75] is used throughout PETSc's interface to perform direct solves. With this solver the matrices are reordered using a quotient minimal degree (qmd) algorithm [76, 77]. The postprocessing and graphics are generated using ParaView [78] and Matplotlib [79]. Finally, the meshes for the simulations are generated with GMSH [80, 81, 82] and partitioned with METIS $[68,83]$.

\subsection{Impact onto a metal plate with rounded cutouts}

The first example is an impact problem presented in Fig. 3a. Consider a square plate of size $100 \mu \mathrm{m}$ with square cutouts of $25 \mu \mathrm{m}$ at the top-right and bottomleft corners. The corners of the cutouts are rounded with concave fillets of radii $R=3 \mu \mathrm{m}$ to avoid too large stress concentrations in those areas. The bottom and right edges of the plate are fixed while the top edge is subjected to an impact velocity $v_{B C}$ that results in large compressive stresses in the plate. The applied velocity profile is shown in Fig. 6. At time $t=0 \mu \mathrm{s}, v_{B C}=0 \mathrm{~m} / \mathrm{s}$ and then it increases linearly to $v_{B C}=25 \mathrm{~m} / \mathrm{s}$ at time $t=2 \mu \mathrm{s}$ after which it remains constant. The initial applied temperature on the plate is uniform and set to $T=293 \mathrm{~K}$, with the normal thermal flux on all edges of the plate being null i.e. insulated conditions. Under this assumption the heat generated by plastic deformations remains in the plate during the simulation.

The flow law used in this example is a modified Litonski law [84] which has the following expression

$$
g\left(\bar{\sigma}, T, \bar{\gamma}_{p}\right)=\dot{\bar{\gamma}}_{p, r e f}\left[\frac{\bar{\sigma}}{\sigma_{r e f}\left\{1+\bar{\gamma}_{p} / \bar{\gamma}_{p, r e f}\right\}^{N}\left\{1+\delta_{t h}-\delta_{t h} \exp \left(\frac{T-T_{r e f}}{k}\right)\right\}}\right]^{m} .
$$

The four parameters $T_{r e f}, \sigma_{r e f}, \bar{\gamma}_{p, r e f}$ and $\dot{\bar{\gamma}}_{p, r e f}$ are the reference temperature, the yield stress and strain and the reference strain rate. $N$ and $m$ are the strain hardening and rate sensitivity exponents and $\delta_{t h}$ and $k$ are thermal softening parameters. Material parameters used for this example are reported in Table B.10 of Appendix B.

In this example the geometry leads to a natural concentration of stresses at the two opposite fillets and hence no imperfection is required to trigger the shear band. Snapshots of the equivalent plastic strain field at time $t=0.30 \mu \mathrm{s}, t=0.39 \mu \mathrm{s}$ and $t=0.54 \mu \mathrm{s}$ are plotted on the deformed configurations and shown in Fig. 7. It can 

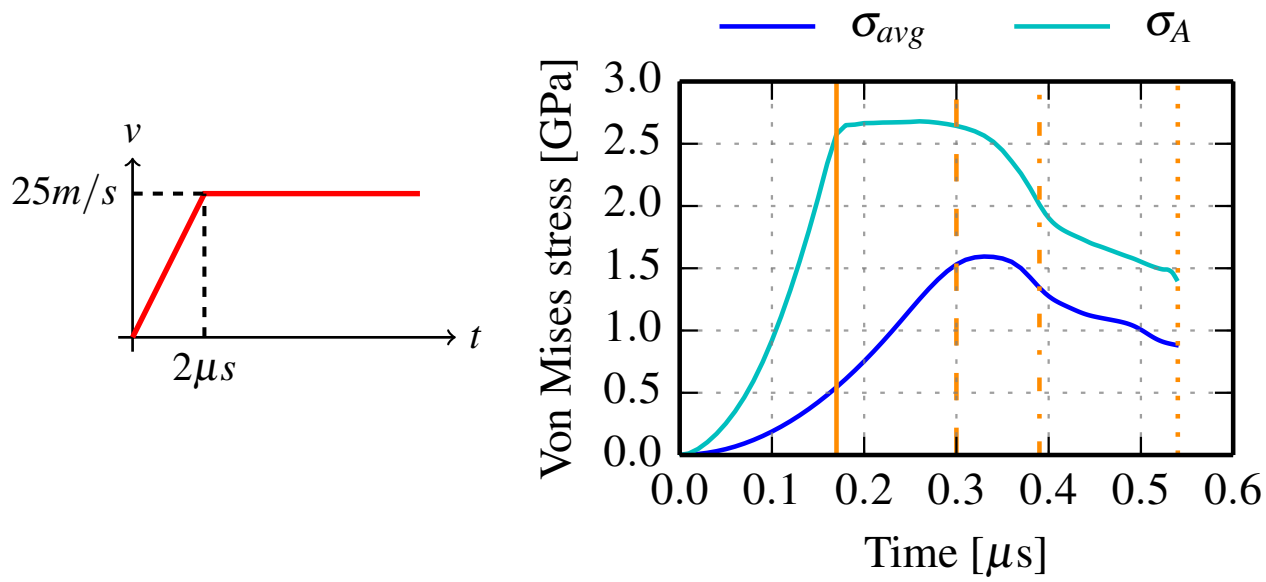

Figure 6: Impact velocity profile applied to the top edge of the plate presented in Fig. $3 \mathrm{a}$ and shown on the left. Average $\sigma_{\text {avg }}$ and local stress at point A, $\sigma_{A}$ shown on the right. The solid orange vertical line - marks the onset of plasticity, the dashed orange vertical line -- marks the onset of thermal softening, the dash-dotted orange vertical line - - marks the stress collapse regime and the dotted orange vertical line - marks the end of the simulation. Note that the dashed lines correspond to the times shown in Fig. 7.
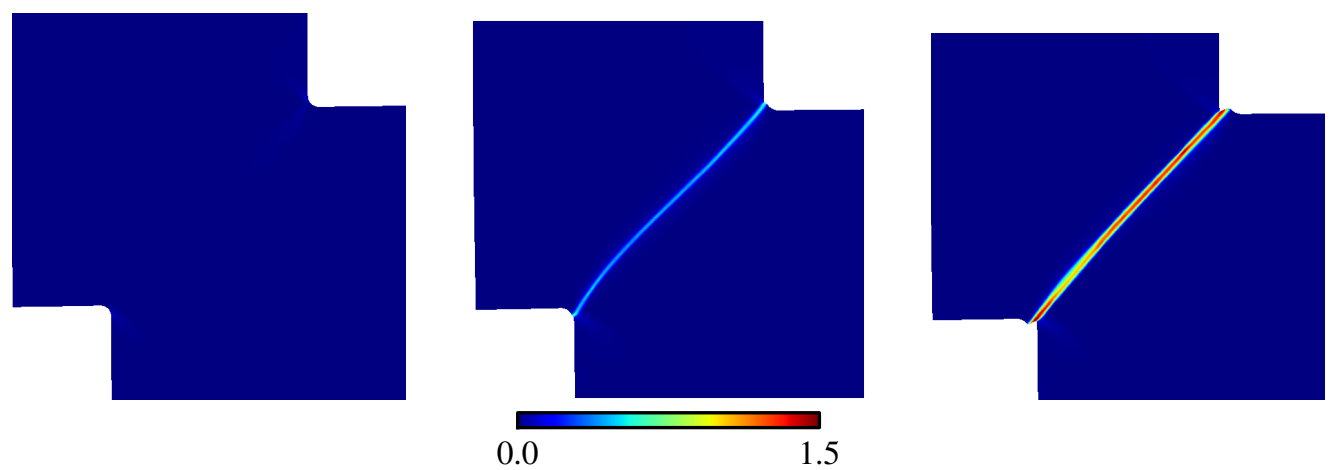

Eq. Plastic Strain

Figure 7: Development of a shear band along the diagonal of an impacted steel plate. Plots of the equivalent plastic strain field on the deformed configuration are illustrated at three times: $t=0.3 \mu \mathrm{s}, t=0.39 \mu \mathrm{s}$ and $t=0.5 \mu \mathrm{s}$ (from left to right). These times are marked on the stress profiles of Fig. 6 by dashed, dash-dot and dotted orange vertical lines, respectively. 
be seen that the shear band extends from one fillet to the other and that a significant displacement jump occurs along the band.

Three unstructured meshes with quadrilateral elements are considered in the simulations, two of which (coarsest and finest) are depicted in Fig. 8. A local refinement along the axis of the shear band is used to ensure that the shear band features are resolved accurately. Note that the plots in Fig. 7 are obtained on the fine mesh. Finally for all the simulations presented in subsection 4.2 and subsection 4.3 the width of the shear band domain is set to $5 \mu \mathrm{m}$.
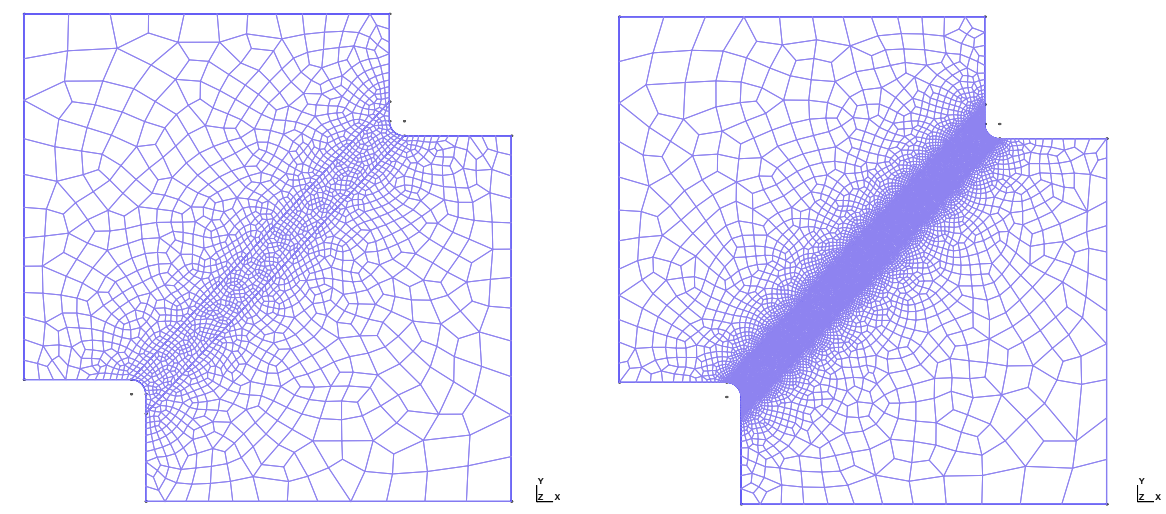

Figure 8: 2,173 nodes coarse mesh (left) and 10,084 nodes fine mesh (right) used for the plate impact problem with local refinement along the shear band.

\subsection{Influence of the overlap length}

In this section we study the effect of the overlap domain length and strategy on the convergence of both $\mathrm{ASM}_{u}$ and ASM Domain Decomposition type preconditioners. Fig. 3c and Fig. 3d show a simplified illustration of the approach used to set the overlapping domains. Without loss of generality and in order to keep the analysis of the results simple, the values for $\delta_{h}$ and $\delta_{s b}$ are set to a constant value: $\delta$ or 0 . A finer approach would be to set independently the values of $\delta_{h}$ and $\delta_{s b}$.

Table 1 summarizes the number of nodes in the healthy and shear band subdomains and in the resulting overlaps for varying values of $\delta$. The number of nodes in the overlap region of $\Omega_{h}$ increases rapidly as the overlaps extend in the most refined region of the mesh whereas the number of nodes in the overlap region of $\Omega_{s b}$ increases more steadily. For these simulations no updates of $\mathbf{S}_{h}^{\text {lin }}$ were necessary and hence the preconditioner takes the form given in Eq. 31. 


\begin{tabular}{c|ccc}
$\delta$ & $1 \mu m$ & $2 \mu m$ & $4 \mu m$ \\
\hline Nodes in $\delta_{h}$ & 505 & 1164 & 2704 \\
Nodes in $\delta_{s b}$ & 367 & 622 & 976
\end{tabular}

Table 1: Number of nodes in each domain for varying overlap sizes $\delta$. The total number of nodes in the mesh is 5463 , the number of nodes in $\Omega_{h}$ is 1969 and the number of nodes in $\Omega_{s b}$ is 3494 .

As observed in Fig. 9, the convergence of both preconditioners is significantly improved when the size of the overlap region grows. The reduction in iterations for the $\mathrm{ASM}_{u}$ preconditioner is well correlated with the length of the overlap domain. The ASM preconditioner experiences a similar behaviour except for $\delta=2 \mu \mathrm{m}$ which requires more linear iterations than the case of $\delta=1 \mu \mathrm{m}$. That behavior is due to the fact that, at time $t=0.2 \mu \mathrm{s}, 11$ Newton iterations are required for $\delta=2 \mu \mathrm{m}$ which triggers a reduction of the time step size, whereas for $\delta=1 \mu \mathrm{m}$ only 10 Newton iterations are required for convergence and no time step reduction takes place. It is interesting to note that this behavior of the numerical algorithm happens at the onset of plasticity as marked on Fig. 6. Another interesting point to observe is that a change of slope on Fig. 9 for the $\mathrm{ASM}_{u}$ preconditioner happens at $t=0.4 \mu \mathrm{s}$ which corresponds to the stress collapse marked on Fig. 6. These observations clearly show how the physical behavior of the solid impacts the convergence of the preconditioner.

Another overlapping possibility is to allow only one of the subdomains to have an overlap. This leads to a lighter preconditioner since one of the subdomain matrices will be smaller. However, it may also yield slower convergence rates [65]. Using the same mesh used to assess the importance of the overlap length, we first set $\left(\delta_{h}, \delta_{s b}\right)=(\delta, \delta)$, then $\left(\delta_{h}, \delta_{s b}\right)=(0, \delta)$ and finally $\left(\delta_{h}, \delta_{s b}\right)=(\delta, 0)$ for $\delta=1 \mu \mathrm{m}, 2 \mu \mathrm{m}$ and $4 \mu \mathrm{m}$. In this experiment, only the $\mathrm{ASM}_{u}$ preconditioner is used. The CPU time and linear iteration count results are reported in Table 2 and Fig. 10 respectively, for a simulation time of $5 \mu \mathrm{s}$.

As expected, in Table 2 we observe that the CPU time is strongly correlated with the number of nodes in the overlap regions. Note that in the case of $\left(\delta_{h}, \delta_{s b}\right)=(0, \delta)$, an overlap length of $\delta=4 \mu \mathrm{m}$ reduces the computational time compared with an overlap length of $\delta=2 \mu \mathrm{m}$. This is due to the large amount of iterations required at the onset of plasticity for $\delta=2 \mu \mathrm{m}$.

From Fig. 10 it can be observed that despite the better performance in term of CPU time of the smaller overlaps, those are yielding significantly larger number of iterations. Moreover, it is also interesting to note that not all overlapping 

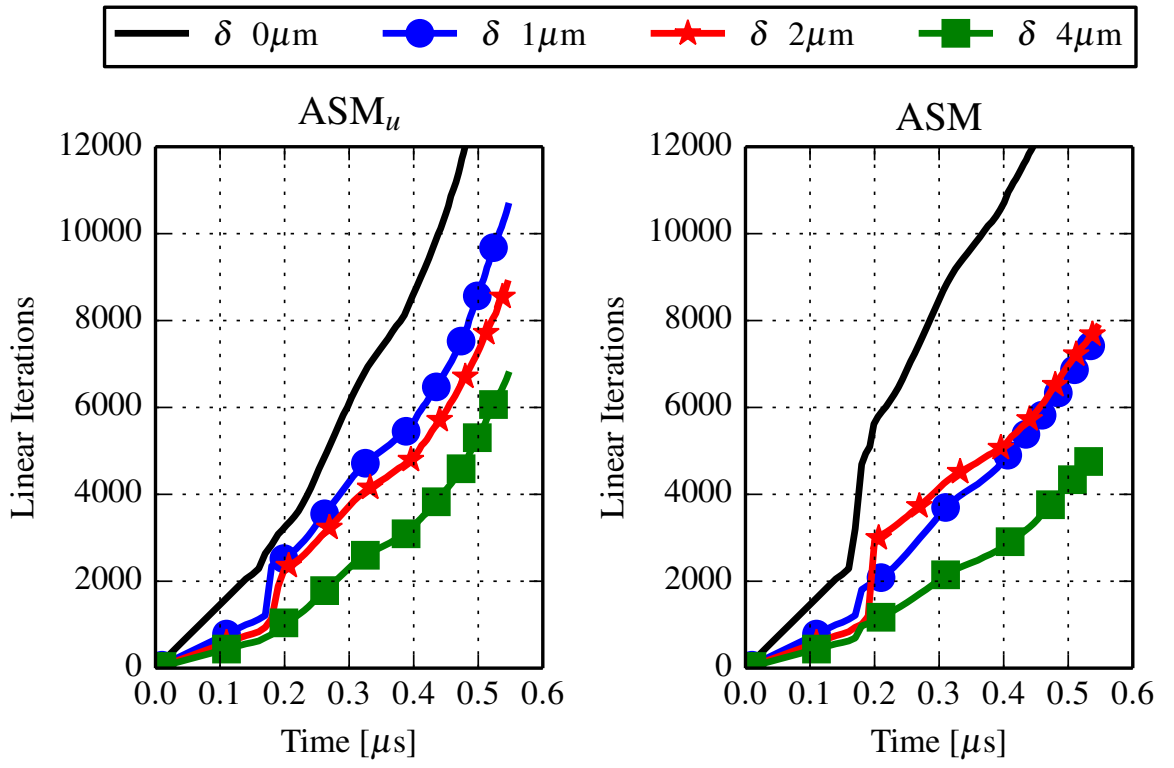

Figure 9: Cumulated number of linear iterations required for the $\mathrm{ASM}_{u}$ (left) and ASM (right) preconditioners to converge for different overlapping lengths.

\begin{tabular}{c|ccc}
$\delta[\mu m]$ & $\left(\delta_{h}, \delta_{s b}\right)=(\delta, \delta)$ & $\left(\delta_{h}, \delta_{s b}\right)=(\delta, 0)$ & $\left(\delta_{h}, \delta_{s b}\right)=(0, \delta)$ \\
\hline 1 & 812.59 & 798.28 & 773.77 \\
2 & 899.79 & 839.89 & 786.15 \\
4 & 987.42 & 847.35 & 778.97
\end{tabular}

Table 2: CPU time in seconds recorded after $5 \mu \mathrm{s}$ simulation of a shear band formation in a plate impact problem, for three overlapping Domain Decomposition strategies and the proposed $\mathrm{ASM}_{u}$ preconditioner. 
schemes give good iteration counts. For example, when $\left(\delta_{h}, \delta_{s b}\right)=(\delta, 0)$, the healthy overlap subdomain $\delta_{h}$ contains more nodes than $\delta_{s b}$ yet its performance is not optimal. This counterintuitive result has to do with the assumption that this subdomain remains nearly unaffected by the shear band during the analysis, which is clearly not the case as large plastic deformation occur within this subdomain. Hence, in order to use the aforementioned Domain Decomposition scheme to accelerate the convergence, the subdomain should be properly selected. This is to say that the healthy subdomain overlap, $\delta_{h}$, should be small to avoid having it extend into the plastic zone and the shear band subdomain overlap, $\delta_{s b}$, can be comparatively large since it typically extends in a region that is not refined.

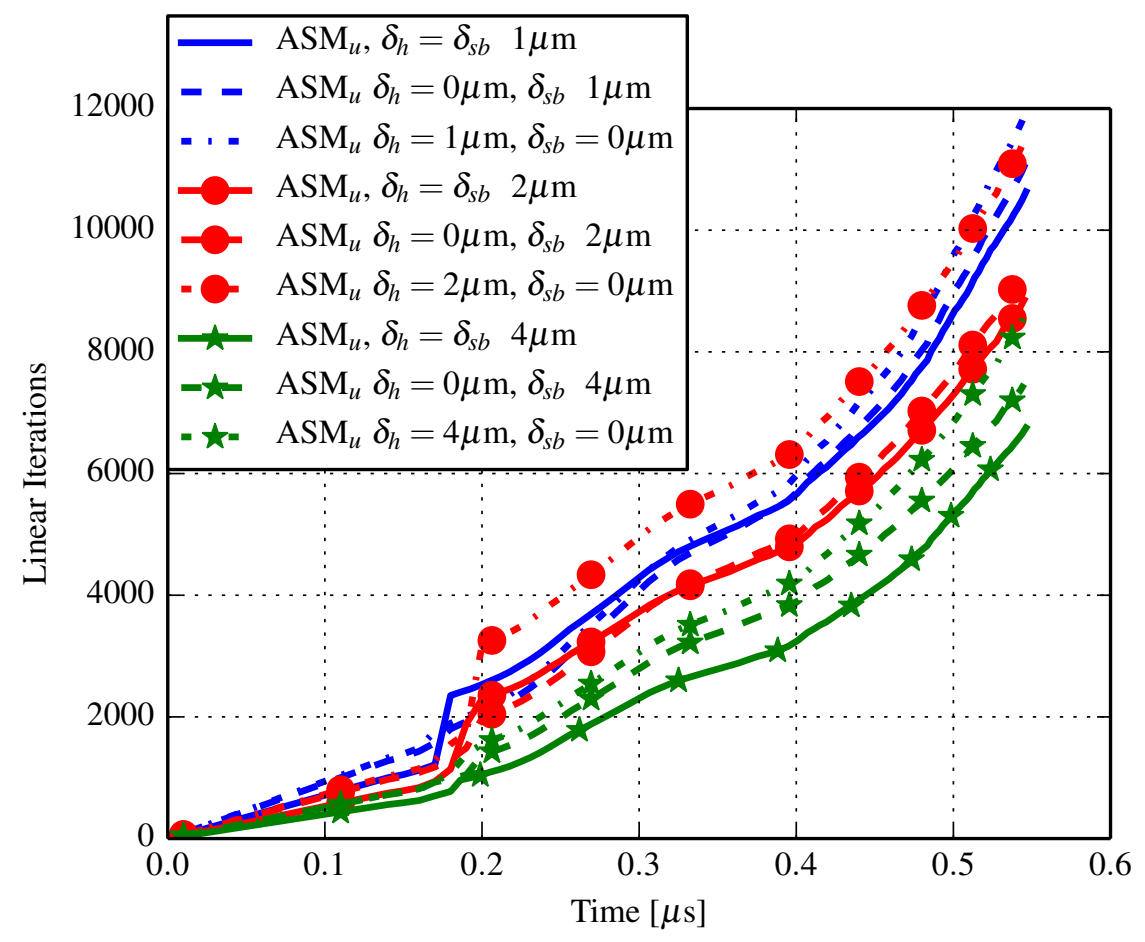

Figure 10: Total number of linear iterations required to compute $0.5 \mu s$ of simulation.

\subsection{Preconditioner performance upon mesh refinement}

In this example, three different levels of mesh refinement in the central region of the plate (where the shear band is expected to form), are considered. The size 
of the overlap between the two subdomains is set to $\delta=1 \mu \mathrm{m}$, and fixed for all meshes. The meshes, subdomains and overlap information for all the cases studied are presented in Table 3.

Remark 4: As the mesh is refined the number of nodes in the overlap region increases and hence lower number of iterations of the preconditioned GMRES algorithm is expected, as shown for the example in Fig. 9.

\begin{tabular}{c|ccccc} 
Mesh & Nodes & Healthy & Healthy overlap & Shear band & Shear band overlap \\
\hline Coarse & 2173 & 1257 & 165 & 916 & 132 \\
Medium & 5463 & 1969 & 505 & 3494 & 367 \\
Fine & 10084 & 2500 & 1022 & 7584 & 584
\end{tabular}

Table 3: Summary of the meshes, domains and overlap information for the three cases studied.

The simulation wall time results associated with each solver-preconditioner pair for all the meshes are summarized in Table 4. Note that no updates of $\mathbf{S}_{h}^{\text {lin }}$ are triggered in this analysis, which means that the preconditioner reduces to the one described in Eq. 31.

It can be observed from Fig. 6 that the plasticity starts at $t=0.17 \mu \mathrm{s}$. This translates to a jump in the number of linear iterations and a change of slope for the number of nonlinear iterations, see Fig. 11. One can also observe that the global stress collapse which starts at around $t=0.37 \mu \mathrm{s}$ corresponds to a second change of slope in the curves depicting the number of linear and nonlinear iterations. This further confirms that the physical process has a strong effect on the nonlinear and linear solvers through a change in the deformation state that eventually leads to a change of spectral radius of the linearized system of equations.

\begin{tabular}{c|ccccc} 
Mesh & $\mathrm{LU}(\mathbf{J})$ & $\mathrm{LU}(\mathbf{S})$ & $\mathrm{GMRES}(\mathbf{S}, \mathrm{ILU}(0))$ & $\mathrm{ASM}$ & $\mathrm{ASM}_{u}$ \\
\hline Coarse & 373.36 & 401.91 & 399.93 & 401.31 & 332.81 \\
Medium & 1272.19 & 1260.48 & 1256.41 & 1242.89 & 1034.71 \\
Fine & 2909.45 & 2715.76 & 5015.37 & 1947.29 & 1828.12
\end{tabular}

Table 4: CPU times (in seconds) for modeling shear band formation under impact for three different meshes corresponding to the problem in subsection 4.1 . 
On all meshes, the proposed $\mathrm{ASM}_{u}$ preconditioner outperforms the other solvers in terms of simulation time. It is closely followed by the ASM preconditioner which provides almost as good CPU timings.

The ILU(0) preconditioner performs very well on the coarsest mesh but its scaling is very poor. Furthermore, as expected, the $\mathrm{LU}(\mathbf{J})$ and $\mathrm{LU}(\mathbf{S})$ solvers have the worst scalings, with $\mathrm{LU}(\mathbf{J})$ performing slightly better than $\mathrm{LU}(\mathbf{S})$ on the coarsest mesh because of the time associated with computing the Schur complement. Nonetheless, forming the Schur complement proves to be a good option as the mesh is refined and $\mathrm{LU}(\mathbf{S})$ outperforms $\mathrm{LU}(\mathbf{J})$.

The total number of nonlinear iterations required for the ASM and $\mathrm{ASM}_{u}$ preconditioners on all the meshes (presented in Fig. 11) is similar. This justifies the reuse strategy we propose in this work for the healthy domain. We also notice that the $\mathrm{LU}(\mathbf{S})$ solver requires a number of nonlinear iterations that is close to those obtained by the proposed preconditioners.

In contrast to that, the GMRES-ILU(0) preconditioner performance is not reported in Fig. 11 as it requires too many Newton iterations to converge. The GMRES-ILU(0) often reaches the maximum number of iterations allowed for the solution of a given linearized system which leads to time step size reductions and hence more Newton iterations compared to the other proposed solvers.

Fig. 11 presents the number of linear iterations required by the $\mathrm{ASM}_{u}$ and ASM preconditioners on three different meshes. The first trend noticed is that the ASM preconditioner always outperforms the $\mathrm{ASM}_{u}$ preconditioner, which is expected since ASM updates the preconditioner of the healthy domain at every time step. Surprisingly though the $\mathrm{ASM}_{u}$ has a better convergence on the fine mesh until time $t=0.42 \mu \mathrm{s}$, after which the ASM catches up.

After investigation it appears that this is due to the fact that when the plasticity starts to develop in the plate the ASM preconditioner is slightly slower than $\mathrm{ASM}_{u}$ which leads to a time step reduction and hence more linear iterations. This phenomenon is not indicative of the convergence of ASM compared to $\mathrm{ASM}_{u}$ but more of an artifact of the specific value of the convergence criteria for this experiment.

The second trend is that despite the fact that the $\mathrm{ASM}_{u}$ preconditioner requires more linear iterations than the ASM preconditioner, the two still give similar CPU wall times due to the much less expensive approach of $\mathrm{ASM}_{u}$.

Finally in Fig. 12 we show the cumulated number of linear iterations of the GMRES(S,ILU(0)) preconditioner compared to the $\mathrm{ASM}_{u}$ and ASM preconditioners. The cumulated number of linear iterations for the GMRES(S,ILU(0)) 

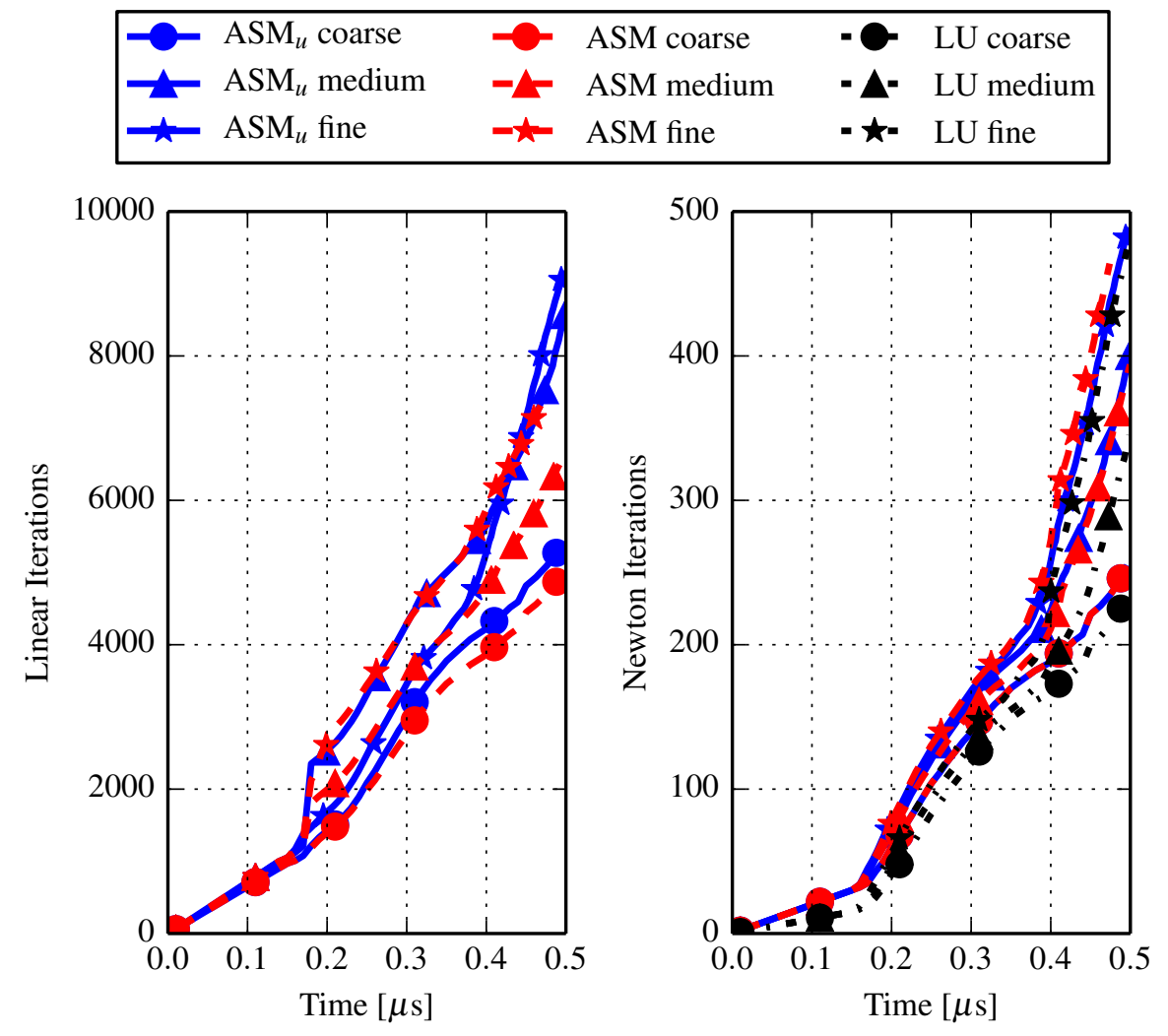

Figure 11: Performance of the preconditioners in terms of linear (left) and nonlinear (right) iterations as function of mesh refinement. 
preconditioner is much larger than that required by the other preconditioners. This illustrates well the fact that the shear band problem is a difficult problem as the physics changes with the deformation and the number of iterations blows up in the softening regime while being reasonable at the onset of plasticity.

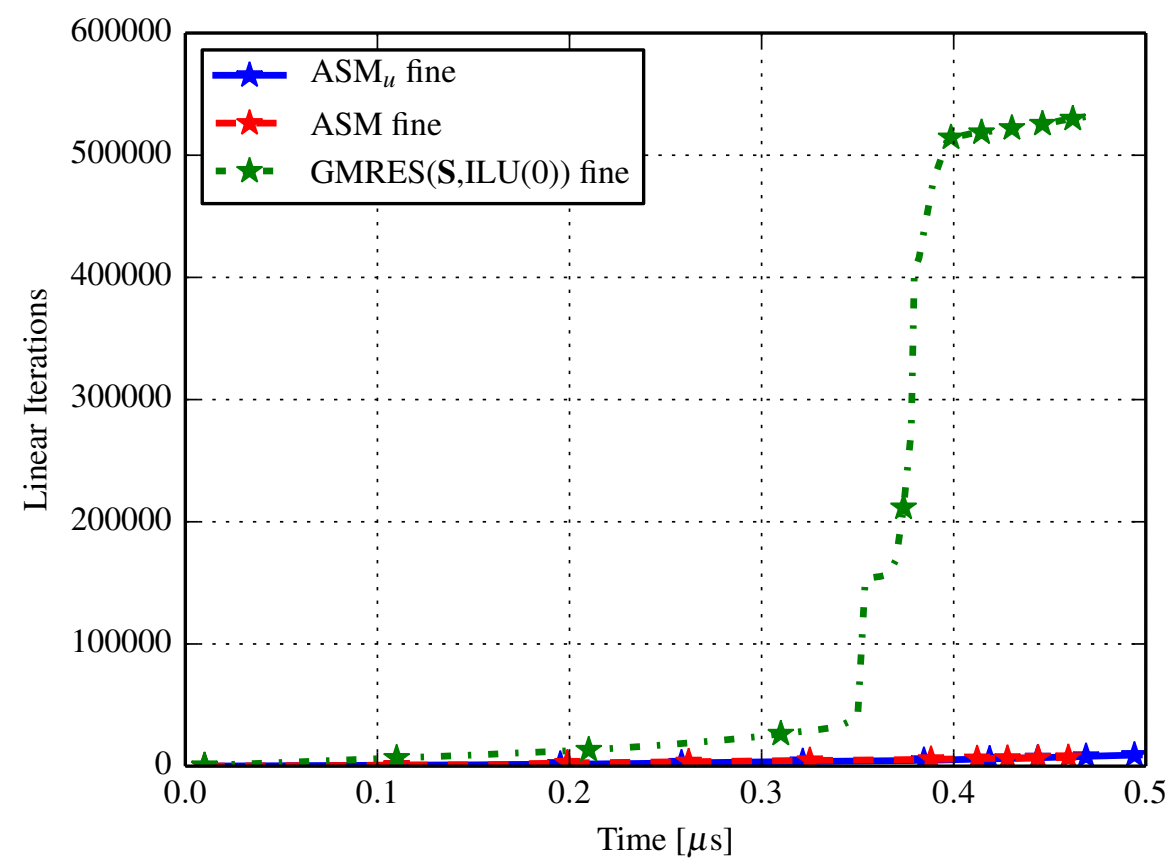

Figure 12: Comparison of the cumulated number of linear iterations for the three GMRES based solvers on the fine mesh.

Remark 5: The change of slope after $t=0.18 \mu \mathrm{s}$, which marks the onset of plastic deformations in the solid can clearly be seen in Fig. 11. At time $t=0.39 \mu \mathrm{s}$, a second change of slope can be observed, this time is due to the beginning of stress collapse in the solid.

\subsection{Preconditioner performance on a $45^{\circ}$ shear band problem using Isogeomet- ric discretization \\ In this section we study the performance of the overlapping Domain Decom- position preconditioner on a shear band benchmark problem with the equations}


discretized using Isogeometric type elements. Isogeometric Analysis leads to denser Jacobian matrices and it is therefore important to study the proposed Domain Decomposition approach on such systems.

Consider a square metal plate under high strain rate tension leading to the formation of a shear band at $45^{\circ}$. In this problem the shear band is triggered by a central imperfection, in which reduced values of yield stress and yield strain are imposed by a $2 \mathrm{D}$ beta function, as proposed in [30], and given as

$$
\sigma_{\text {yield }}=\sigma_{0} \beta_{2 D}(x, y), \quad \gamma_{\text {yield }}=\gamma_{0} \beta_{2 D}(x, y)
$$

with

$$
\beta_{2 D}(x, y)=1-0.04\left[\operatorname{sech}\left(\frac{\sqrt{x^{2}+y^{2}}}{10^{-5}}\right)\right]^{2} .
$$

This corresponds to a smoothed imperfection centered at $(x, y)=(0,0)$ with a maximal reduction factor of $4 \%$. Under these assumptions, an X-shaped shear band forms in the plate but due to symmetry considerations only a quarter of the plate (top-right quarter) is modeled, as illustrated in Fig. 13a. The plate dimensions, boundary conditions and loading are illustrated in Fig. 13a. Note that the applied loading increases linearly from $v_{B C}=0 \mathrm{~m} / \mathrm{s}$ at $t=0 \mu \mathrm{s}$ to $v_{B C}=5 \mathrm{~m} / \mathrm{s}$ at $t=10 \mu \mathrm{s}$ and $v_{B C}$ remains constant afterwards. The initial displacements, stresses and equivalent plastic strains at time $t=0$ are set to zero and the temperature is set to $T=293 K$ (room temperature) with all fluxes assumed to be null at all times (insulated conditions). Similar to the problem in subsection 4.1, material parameter values are given in Table B.10. The shear band that develops from the bottom-left to the upper-right corners of the plate is shown in Fig. $13 \mathrm{~b}$.

In order to assess the performance of the $\mathrm{ASM}_{u}$ preconditioner on Isogeometric discretizations, we discretize the system in Eq. 1 using the higher order NURBS Shear Band Quadrilateral (INSQ) element (so far only first order INSQ elements have been used, these first order elements are also called ISBQ elements). This element along with other NURBS type elements have recently been developed and studied by Berger-Vergiat et. al [32, 85], the reader is referred to these papers for more information on the discretization and performance of the INSQ element. The overlap strategy used in this section is as follow: $\delta_{h}=0$ and $\delta_{s b}=\delta$ and the width of the shear band domain is $5 \mu \mathrm{m}$.

Herein, two studies are conducted to analyze the convergence of the preconditioner:

I. the problem is solved with a coarse ( 20 by 20 elements) and a fine ( 40 by 


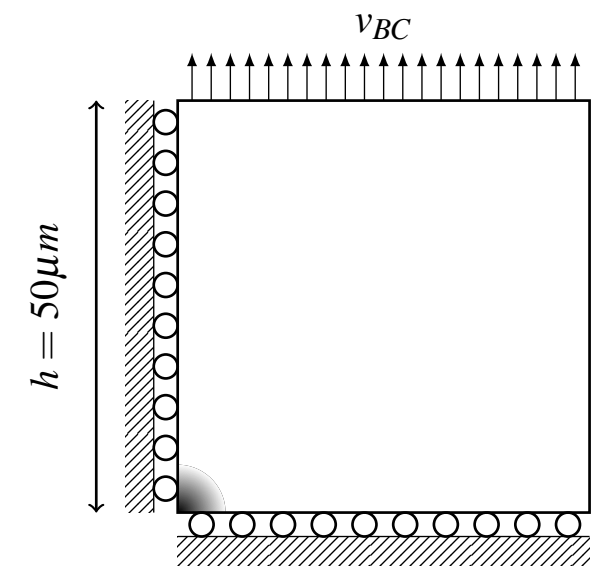

(a) A square plate dimension, boundary conditions and applied velocity $v_{B C}$

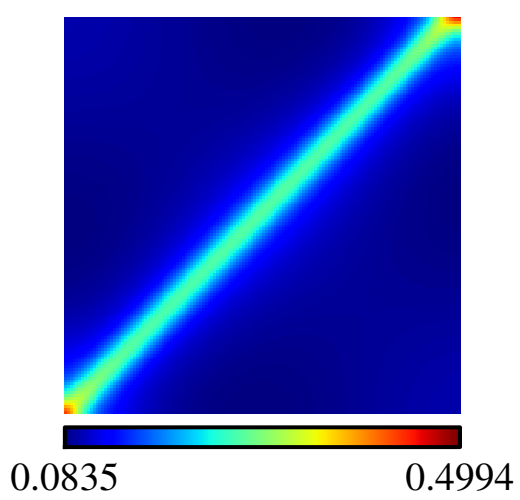

(b) EQPS at final time step

Figure 13: A $45^{\circ}$ shear band benchmark problem with material imperfection at the bottom-left corner. A well developed shear band at the end of Stage 3 deformation state is shown in (b).

40 elements) using shape functions of polynomial order $1,2,3$ and 4 to observe the effect of $h$ - and $k$-refinment,

II. the overlap region is increased from $1 \mu \mathrm{m}$ to $2 \mu \mathrm{m}$ and to $4 \mu \mathrm{m}$ to evaluate the influence of the size of the support of the shape functions on the convergence of the Domain Decomposition method.

In these example cases, no update of $\mathbf{S}_{h}^{\text {lin }}$ is triggered and hence the healthy domain preconditioner is computed only once and reused throughout the entire analysis. Due to the tensorial nature of NURBS based elements, it is easy to find equations that relate the number of elements, nodes, equations and nonzeros per row (for nodes not on a boundary). Such information, reported in Table 5, is directly related to the amount of work necessary to form and solve the linearized system of equations at each Newton iteration.

The CPU timings (in seconds) using Isogeometric Analysis with four different polynomial orders for simulation of the $45^{\circ}$ shear band problem, with 20 by 20 and 40 by 40 elements mesh, are reported in Table 6 and Table 7, respectively. In this study an overlap of $\delta=1 \mu \mathrm{m}$ is considered.

Table 6 shows that on the coarser $20 \times 20$ mesh the ILU(0) preconditioner proves to be the most efficient for NURBS orders $P 1$ to $P 3$ while for denser order $P 4$ the proposed $\mathrm{ASM}_{u}$ is the most efficient. These results mainly show that on 


\begin{tabular}{c|cccc}
$p$ & $\begin{array}{c}\text { Nodes } \\
(n e l+p)^{d}\end{array}$ & $\begin{array}{c}\text { Equations } \\
n d f(n e l+p)^{d}\end{array}$ & $\begin{array}{c}\text { Nonzeros } \\
\text { per row } \\
n d f(2 p+1)^{d}\end{array}$ & $\begin{array}{c}\text { Quadrature } \\
\text { points } \\
{[(p+1) n e l]^{d}}\end{array}$ \\
\hline 1 & 441 & 1323 & 27 & 1600 \\
2 & 484 & 1452 & 75 & 3600 \\
3 & 529 & 1587 & 147 & 6400 \\
4 & 576 & 1728 & 243 & 10000
\end{tabular}

Table 5: Mesh information for Isogeometric Analysis used on the benchmark example in Fig. 13 with $n e l$ elements per edge (here $n e l=20$ ), $p$ order NURBS, $n d f$ degrees of freedom per node (here $n d f=3$ ) and $d$ spatial dimensions (here $d=2$ )

small meshes the GMRES solver is quite efficient and that an off-the-shelf ILU(0) preconditioner is sufficient to accelerate it. One can also observe that $\mathrm{ASM}_{u}$ and $\mathrm{LU}(\mathbf{S})$ have close performance especially on higher order discretization which further indicates that on this small mesh most solution methods perform similarly well.

On the $40 \times 40$ mesh, for which results are reported in Table 7 , the proposed $\mathrm{ASM}_{u}$ and ASM algorithm show superior performance with the increase of the discretization order, $p$. This is further emphasized by the fact that on the $20 \times 20$ mesh the $\mathrm{ASM}_{u}$ preconditioner was only $2 \%$ faster than the LU(S) whereas it is $9 \%$ faster on the $40 \times 40$.

Overall the results of this study show that the proposed preconditioner provides excellent convergence behaviour for higher order discretizations and also provides better efficiency on larger meshes.

\begin{tabular}{c|ccccc} 
Order & $\mathrm{LU}(\mathbf{J})$ & $\mathrm{LU}(\mathbf{S})$ & $\mathrm{ILU}(0)$ & $\mathrm{ASM}$ & $\mathrm{ASM}_{u}$ \\
\hline P1 & 31.71 & 30.58 & 30.51 & 32.82 & 33.62 \\
P2 & 105.40 & 89.07 & 84.48 & 92.92 & 88.97 \\
P3 & 315.05 & 254.55 & 251.24 & 253.56 & 252.48 \\
P4 & 799.05 & 638.55 & 646.20 & 652.25 & 626.40
\end{tabular}

Table 6: CPU times in seconds for the simulation of the $45^{\circ}$ shear band problem using Isogeometric discretization for four different polynomial orders on a 20 by 20 elements mesh

Next, we study the influence of the overlap length on the convergence of the 


\begin{tabular}{c|ccccc} 
Order & $\mathrm{LU}(\mathbf{J})$ & $\mathrm{LU}(\mathbf{S})$ & $\mathrm{ILU}(0)$ & $\mathrm{ASM}$ & $\mathrm{ASM}_{u}$ \\
\hline P1 & 131.41 & 123.68 & 121.16 & 144.05 & 138.22 \\
P2 & 483.72 & 396.64 & 549.76 & 412.43 & 392.63 \\
P3 & 1302.84 & 1125.47 & 1197.56 & 1121.72 & 1121.79 \\
P4 & 3402.78 & 2815.97 & 2998.29 & 2665.29 & 2563.19
\end{tabular}

Table 7: CPU times in seconds for the simulation of the $45^{\circ}$ shear band problem using Isogeometric discretization for four different polynomial orders on a 40 by 40 elements mesh

preconditioner considering four different overlap length: $\delta=0 \mu m, \delta=1 \mu m$, $\delta=2 \mu \mathrm{m}$ and $\delta=4 \mu \mathrm{m}$ on a 40 by 40 elements mesh discretized with first order (P1) and fourth order (P4) NURBS shape functions.

The cumulative number of linear iterations as a function of the simulation time is presented in Fig. 14 (top). In addition we define an overlap efficiency measure as

$$
\eta_{\text {overlap }}=1-\frac{\text { Iter }_{\delta}}{\operatorname{Iter}_{\delta=0}}
$$

where Iter $\delta$ and Iter $_{\delta=0}$ are the number of iterations required to achieve convergence when an overlap lenght of $\delta \mu m$ or $0 \mu m$ are used. The efficiency defined in Eq. 35 is plotted in Fig. 14 (bottom). This measure shows that for standard P1 discretizations, increase in overlap length clearly leads to reduction in number of iterations. The larger the overlap length, the greater the drop in iterations. However, for P4 discretizations, the situation is far less obvious. At the first stages of deformation, before the stress collapse, increase in overlap indeed leads to reduction in iterations. However after stress collapse the efficiency of the overlap significantly decreases, in particular for the larger overlaps. These results are likely related to the long span interactions of high order NURBS and will be further explored in future research.

\subsection{Parallel results on a Kalthoff notched plate impact example}

In this final numerical example the scalability of the $\mathrm{ASM}_{u}$ preconditioner is investigated on a parallel computer and compared to the aforementioned $\mathrm{LU}(\mathbf{S})$ solver. The study is conducted on a variant of the Kalthoff-Winkler problem [86, 14], previously presented in [87]. The original Kalthoff experiment is depicted in Fig. 15 along with the variant used for our scaling study. Hence, we are primary concerned with the shear band initiation from the notched plate tip. The material properties used for this problem are those of C-4340 steel, presented in Table B.11, 

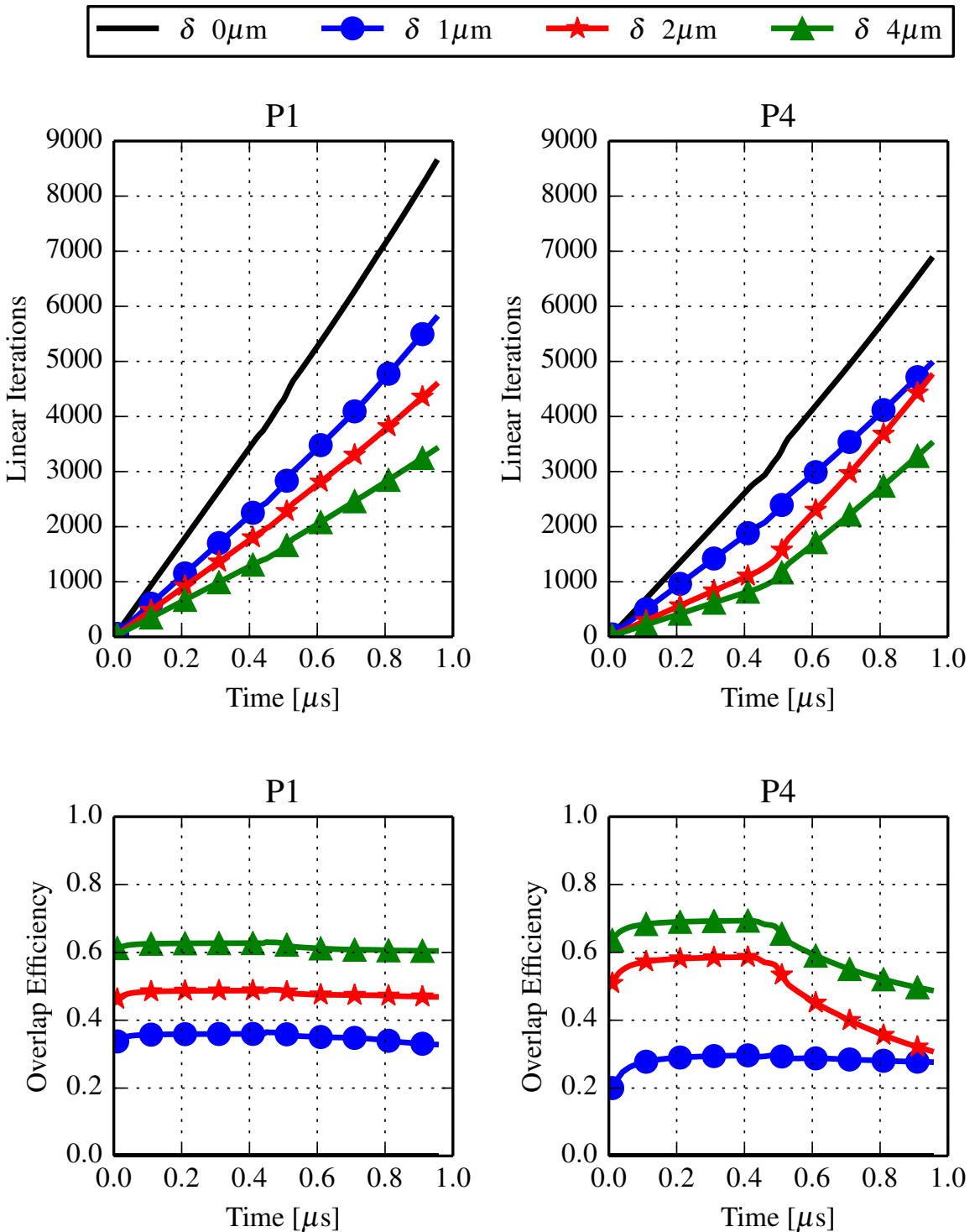

Figure 14: Comparison of the convergence of the $\mathrm{ASM}_{u}$ preconditioner on the 40 by 40 mesh with first (P1) and fourth (P4) order element Isogeometric discretizations of the $45^{\circ}$ shear band problem and various overlap lengths. 


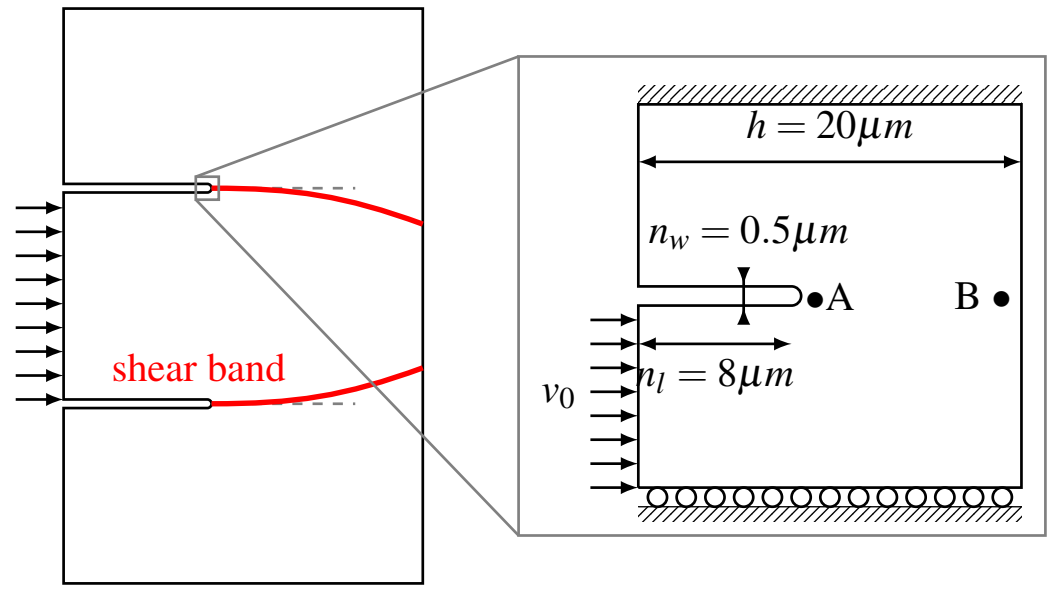

Figure 15: Original Kalthoff experiment (left) and proposed numerical simulation (right).

with a Johnson-Cook flow law that has the following form

$$
\bar{\sigma}=\left(A+B \bar{\gamma}_{p}^{n}\right)\left(1+C \ln \left[\frac{\dot{\bar{\gamma}}_{p}}{\dot{\bar{\gamma}}_{p, 0}}\right]\right)\left(1-\frac{T-T_{\text {room }}}{T_{\text {melt }}-T_{\text {room }}}\right)^{m}
$$

where $A$ is the initial yield stress, $B$ the strain sensitivity, $n$ the strain sensitivity exponent, $C$ the strain rate sensitivity, $\dot{\bar{\gamma}}_{p, 0}$ is the reference equivalent plastic strain rate and $m$ the thermal softening exponent. $T_{m e l t}$ and $T_{\text {room }}$ are the melting and room temperature respectively.

One interesting feature of this example is that it results in a shear band that initiates at the notch tip and then propagates to the right edge of the plate. In contrast to the previously presented examples for which the shear band forms across the plate instantaneously, in this example propagation of the shear band can be clearly observed in Fig. 16. Another interesting feature is the secondary zone of plastic deformation that appears on the diagonal that starts at the notch and extends to the bottom left corner of the plate. This zone has a strong influence on the number of updates to $\mathbf{S}_{h}^{u}$ occuring in the healthy subdomain $\Omega_{h}$ and hence on the rate of convergence of the GMRES iterative solver. The shear band subdomain for this example is simply defined as a band of width $w=0.5 \mu \mathrm{m}$ centered on the notch and extends from the left edge all the way to the right edge of the plate.

First, a strong scaling study is conducted using a problem size of 75,000 equations on which the proposed preconditioner is tested and compared to $\mathrm{LU}(\mathbf{S})$ 


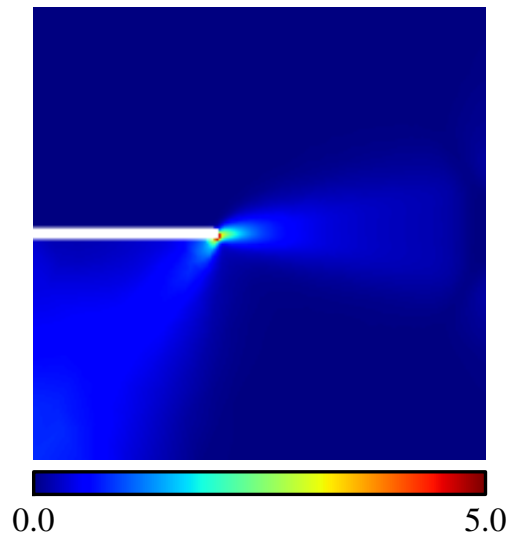

(a) $t=30 \mathrm{~ns}$

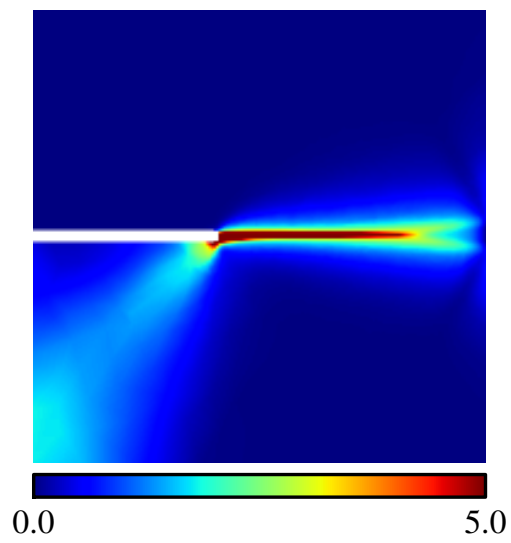

(c) $t=60 n s$

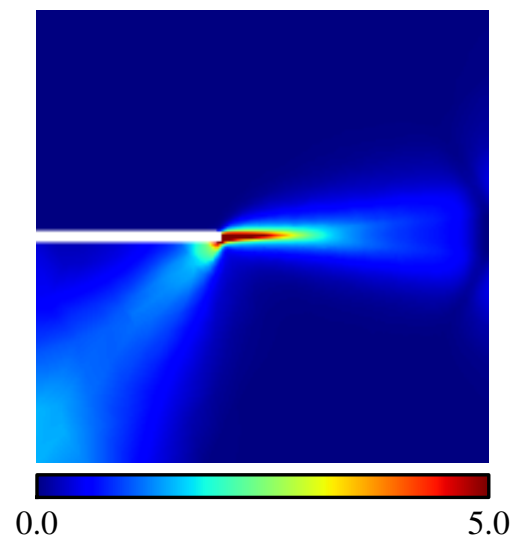

(b) $t=45 n s$

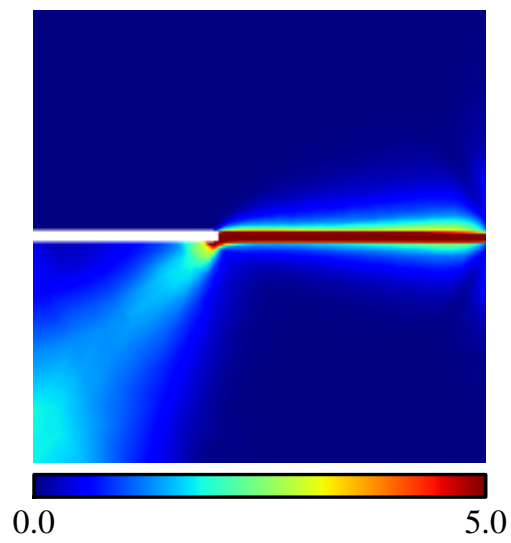

(d) $t=90 \mathrm{~ns}$

Figure 16: Snapshots showing the equivalent plastic strain (EQPS) increasing from the notch tip to the right edge of the plate as the shear band propagates. 
solver. The number of MPI ranks to partition the problem is 1, 2, 4, 8 and 16, each rank is allocated a $1.8 \mathrm{GHz}$ E5-2650L Intel processor. The total CPU times required to solve the problem in all these configurations are reported in Fig. 17. The $\mathrm{ASM}_{u}$ preconditioner required 3, 2, 3, 3 and 2 updates of $\mathbf{S}_{h}^{u}$ when decomposed in 1, 2, 4, 8 and 16 partitions, respectively, which is expected following the plastic zone outside the shear band domain as shown in Fig. 16.

The first observation from these results is that the proposed $\mathrm{ASM}_{u}$ preconditioner performs very well in parallel when compared to the $\mathrm{LU}(\mathbf{S})$ solver. Both CPU Wall time and efficiency of $\mathrm{ASM}_{u}$ are significantly better then those of the $\mathrm{LU}(\mathbf{S})$ solver. Furthermore, it is interesting to note that a favorable load balance is achieved for the $\mathrm{ASM}_{u}$ preconditioner when 2 or 4 processors are used. However when the partitions are too small some processors have nodes mainly in the healthy domain and therefore do not work as much as those processors with nodes mainly in the shear band leading to a decrease in efficiency of the $\mathrm{ASM}_{u}$ preconditioner, eventhough the Wall time keeps on improving. Additional data from the simulation such as number of linear iterations and nonlinear iterations are provided in Table 8

\begin{tabular}{cccccc}
\hline Processors & 1 & 2 & 4 & 8 & 16 \\
\hline $\mathbf{S}_{h}^{u}$ updates & 3 & 2 & 3 & 3 & 2 \\
Linear iterations & 38,762 & 42,444 & 41,216 & 45,622 & 53,819 \\
Walltime [s] & $6,959.32$ & $2,944.68$ & $1,419.48$ & 814.05 & 648.31 \\
\hline
\end{tabular}

Table 8: Simulation data from the strong scaling study of the $\mathrm{ASM}_{u}$ preconditioner. Each simulation required 1563 Newton iterations.

Remark 6: In Fig. 17 it can be observed that the $\mathrm{ASM}_{u}$ preconditioner has an efficiency larger than one when two and four and eight processors are used. This phenomenon is due to the fact that when more than one processor the use of cache memory is performed more efficiently.

Next, we study the weak scaling of the $\mathrm{ASM}_{u}$ preconditioner using successively finer meshes while keeping the number of nodes (and equations) per partition approximately constant. A typical partition obtained with METIS for the 16 partitions case is presented in Fig. 18. It can be observed that the geometric size of the partitions vary with the local node density leading to a domain with more partitions along the shear band refinement zone. 

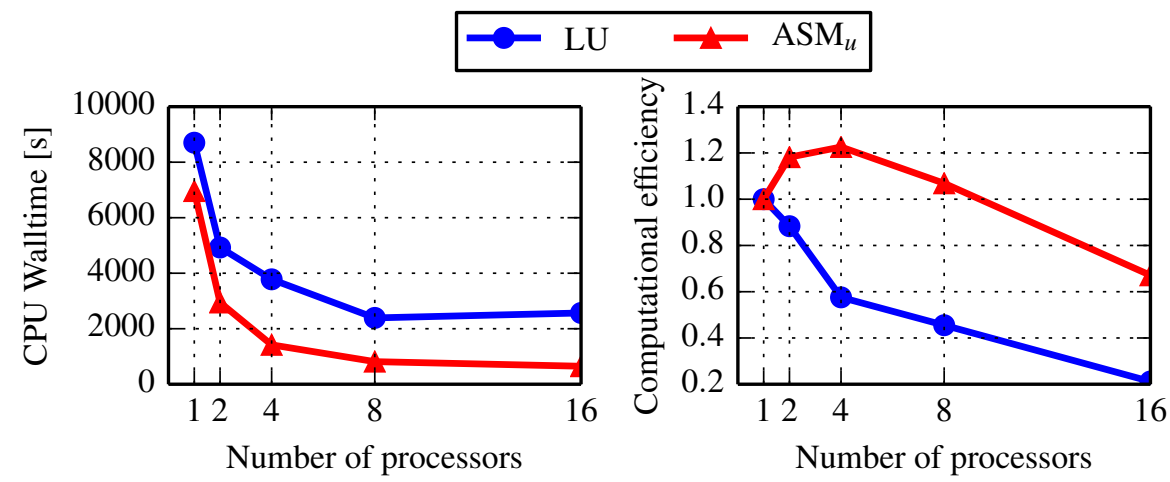

Figure 17: Strong scaling study of the solvers with one processor per partition and one partition per MPI rank. On the left CPU Wall time and on the right efficiency of the preconditioners

A summary of the meshes, number of partitions and number of processors (1.8GHz E5-2650L Intel processor) used for the simulation as well as the overall CPU time required to complete the simulations, is reported in Table 9. It can be observed that the computational time required by the $\mathrm{LU}(\mathbf{S})$ solver is much larger than that required by the $\mathrm{ASM}_{u}$ preconditioner and is also growing at a higher rate, meaning that the $\mathrm{ASM}_{u}$ preconditioner has better weak scaling properties.

Remark 7: From Fig. 18 it is clear that some partitions have more nodes that will be in the shear band domain and other will have more nodes in the healthy domain. This leads to some load balancing issues that can also be seen to some extent in the strong scaling study where ASM scales better since it has the same amount of work on all partitions.

\begin{tabular}{ccccccc}
\multirow{2}{*}{ Nodes } & \multirow{2}{*}{ Equations } & Nodes/partition & Processors & \multicolumn{2}{c}{$\mathrm{CPU}$ time $[\mathrm{s}]$} & $\mathrm{ASM}_{u}$ \\
& & & & $\mathrm{LU}(\mathbf{S})$ & $\mathrm{ASM}_{u}$ & updates \\
\hline 98,017 & 234,051 & 39,008 & 2 & 5,952 & 2,398 & 1 \\
154,621 & 463,863 & 38,655 & 4 & 9,405 & 3,127 & 2 \\
307,744 & 923,232 & 38,468 & 8 & 17,771 & 4,506 & 2 \\
613,285 & $1,839,855$ & 38,350 & 16 & - & 7,456 & 1
\end{tabular}

Table 9: Weak-scaling study on the modified Kalthoff problem with up to 16 processors. 

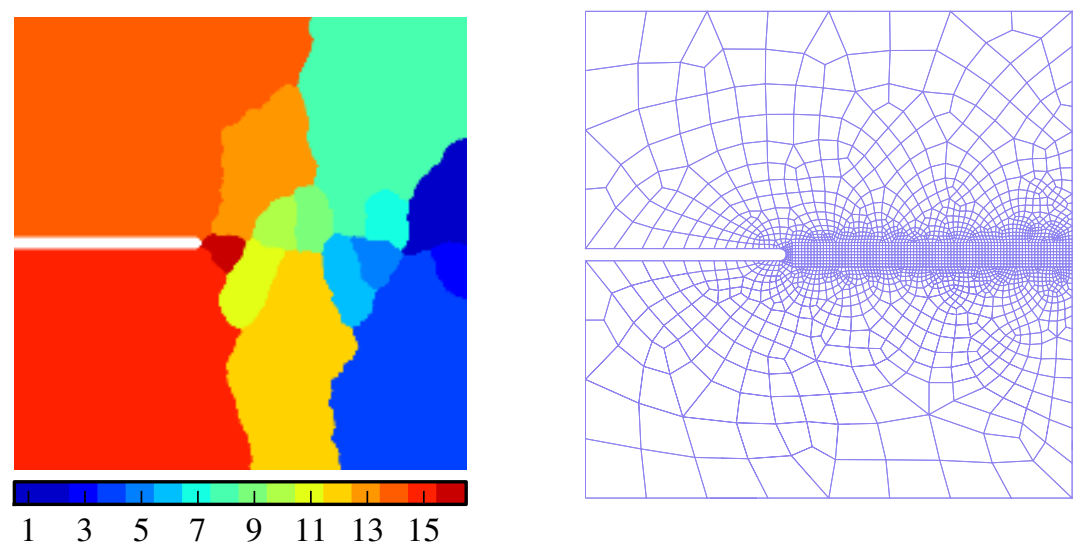

Figure 18: Partitioning of the modified Kalthoff problem discretized with 613,285 nodes into 16 partitions of 38,350 nodes in average (left) and mesh representing the refined area where the shear band develops (right). Note that the depicted mesh is only shown as an illustration but not used for simulations since it is too coarse.

\section{Conclusion}

In this work we develop a field- and domain- decomposition type preconditioner that takes into account the physics and the path of shear band localization. The key idea is to reduce the computational effort required for solving the linearized, monolithic systems by realizing that most of the deformation and plasticity is localized in a narrow band while only minor plasticity happens out of this band.

This assumption allows us to develop preconditioners that concentrate most of the effort in the shear band domain while reusing and selectively updating the information away from the band. This concept is implemented by employing a restricted additive Schwarz algorithm applied as a preconditioner to a GMRES solver for solving the Schur complement of the global Jacobian.

The proposed preconditioner is implemented in serial and parallel and applied to three benchmarks problems. We study standard low order elements as well as high order formulations following Isogeometric Analysis. First we assess the performance of the $\mathrm{ASM}_{u}$ preconditioner as compared to a more expensive version of the same preconditioner ASM that is updated every time step and to a direct LU solver. We conclude that $\mathrm{ASM}_{u}$ gives superior performance.

The influence of the overlapping Domain Decomposition strategy is analyzed for the different Isogeometric discretization orders. While increase in the overlap 
length clearly leads to reduction of linear iterations for low order discretizations, the effect is less obvious for high order discretization that are not compactly supported.

Finally strong and weak scaling studies demonstrate the advantages of the $\mathrm{ASM}_{u}$ preconditioner for parallel computing. In all these studies the preconditioner out-performed state-of-the-practice direct solvers and other preconditioner/GMRES pairs.

\section{Acknowledgment}

The financial support of the Department of Energy through the Early Career Research Program, No. DE-SC0008 196, is gratefully acknowledged.

\section{Appendix A. Additive Schwarz method}

The additive Schwarz method computes an approximate inverse of $\mathbf{S}$ that we will denote $\mathbf{P}_{A S M}^{-1}$, using the successive inverses of $\mathbf{S}_{h}$ and $\mathbf{S}_{s b}$. Hence this method requires both $\mathbf{S}_{h}$ and $\mathbf{S}_{s b}$ to be invertible matrices. Using the following notations

$$
\mathbf{S}_{h}^{-1}=\left[\begin{array}{cc}
\left(\left(\mathbf{S}_{h}\right)_{\mid \Omega_{h}}\right)^{-1} & \mathbf{0} \\
\mathbf{0} & \mathbf{1}
\end{array}\right] \quad \text { and } \quad \mathbf{S}_{s b}^{-1}=\left[\begin{array}{cc}
\mathbf{1} & \mathbf{0} \\
\mathbf{0} & \left(\left(\mathbf{S}_{s b}\right)_{\mid \Omega_{s b}}\right)^{-1}
\end{array}\right],
$$

one iteration of this method between steps $n$ and $n+1$ is derived as follows

$$
\begin{gathered}
\mathbf{r}^{n}=\mathbf{b}-\mathbf{S u}^{n}, \\
\mathbf{u}^{n+1 / 2}=\mathbf{u}^{n}+\mathbf{S}_{h} \mathbf{r}^{n}, \\
\mathbf{u}^{n+1}=\mathbf{u}^{n+1 / 2}+\mathbf{S}_{s b} \mathbf{r}^{n} .
\end{gathered}
$$

an approximate solution to (15) can be computed as

$$
\mathbf{u}=\left[\mathbf{S}_{h}^{-1}+\mathbf{S}_{s b}^{-1}\right] \mathbf{b}=\mathbf{P}_{A S M}^{-1} \mathbf{b} .
$$

Remark 8: Usualy the Schwarz method is used within Richardson or Krylov iterations to obtain a converged value for $\mathbf{u}$ and equation (A.5) is applied to a residual $(\mathbf{r}=\mathbf{b}-\mathbf{S u})$ to compute a correction $\delta \mathbf{u}$.

\section{Appendix B. Material properties}




\begin{tabular}{lccc}
\hline Physical quantity name & Name & Value & SI base unit \\
\hline Young's modulus & $E$ & $200 \mathrm{E} 9$ & $\mathrm{~kg} \cdot \mathrm{m}^{-1} \cdot \mathrm{s}^{-2}$ \\
Poisson's ratio & $v$ & 0.3 & - \\
Mass density & $\rho$ & 7830 & $\mathrm{~kg} \cdot \mathrm{m}^{-3}$ \\
Specific heat & $c_{p}$ & 448 & $\mathrm{~m}^{2} \cdot \mathrm{s}^{-2} \cdot \mathrm{K}^{-1}$ \\
Coefficient of thermal expansion & $\alpha$ & $11.2 e^{-6}$ & $\mathrm{~K}^{-1}$ \\
Thermal conductivity & $\kappa$ & 803.5 & $\mathrm{~kg} \cdot \mathrm{m} \cdot \mathrm{s}^{-3} \cdot K$ \\
Thermal softening parameter & $\delta_{t h}$ & 0.8 & - \\
Thermal softening parameter & $k$ & 500 & $\mathrm{~K}$ \\
Reference temperature & $T_{r e f}$ & 293 & $\mathrm{~K}$ \\
Taylor-Quinney Coefficient & $\chi$ & 0.9 & - \\
Yield stress & $\sigma_{r e f}$ & $2 \mathrm{E} 9$ & $\mathrm{~kg} \cdot \mathrm{m}^{-1} \cdot \mathrm{s}^{-2}$ \\
Yield strain & $\gamma_{r e f}$ & 0.01 & - \\
Reference strain rate & $\dot{\gamma}_{r e f}$ & 0.001 & $\mathrm{~s}^{-1}$ \\
Strain hardening exponent & $\mathrm{N}$ & 0.01 & - \\
Rate sensitivity parameter & $\mathrm{m}$ & 70 & - \\
\hline
\end{tabular}

Table B.10: Material parameters used in the example problems

\section{References}

[1] V. Tvergaard. Influence of voids on shear band instabilities under plane strain conditions. International Journal of Fracture, 17(4):389-407, 1981.

[2] T.W. Wright. The Physics and Mathematics of Adiabatic Shear Bands. Cambridge Monographs on Mechanics. Cambridge University Press, Cambridge, 2002.

[3] A. Marchand and J. Duffy. An experimental study of the formation process of adiabatic shear bands in a structural steel. Journal of the Mechanics and Physics of Solids, 36(3):251 - 283, 1988.

[4] T.W. Wright and J.W. Walter. On stress collapse in adiabatic shear bands. Journal of the Mechanics and Physics of Solids, 35(6):701 - 720, 1987.

[5] K.-M. Cho, S. Lee, S.R. Nutt, and J. Duffy. Adiabatic shear band formation during dynamic torsional deformation of an hy-100 steel. Acta Metallurgica et Materialia, 41(3):923 - 932, 1993. 


\begin{tabular}{cccc}
\hline Name & Notation & Value & Units \\
\hline Density & $\rho$ & 7830 & $\mathrm{~kg} \cdot \mathrm{m}^{-3}$ \\
Young modulus & $E$ & 200 & $\mathrm{GPa}$ \\
Poisson ratio & $v$ & 0.29 & - \\
Thermal conductivity & $\kappa$ & 38 & $\mathrm{~K}$ \\
Thermal capacity & $c_{p}$ & 477 & $\mathrm{~J} \cdot(\mathrm{kg} \cdot \mathrm{K})^{-1}$ \\
Coefficient of thermal expansion & $\alpha$ & $32.0 e^{-6}$ & $\mathrm{~K}^{-1}$ \\
Reference strain rate & $\dot{\varepsilon}_{0}$ & 1.0 & $\mathrm{~s}^{-1}$ \\
Yield stress & $A$ & 792 & $\mathrm{MPa}$ \\
Strain sensitivity & $B$ & 510 & $\mathrm{MPa}$ \\
Strain sensitivity exponent & $n$ & 0.26 & - \\
Strain rate sensitivity & $C$ & 0.014 & - \\
Reference temperature & $T_{\text {room }}$ & 293 & $\mathrm{~K}$ \\
Melting temperature & $T_{m e l t}$ & 1793 & $\mathrm{~K}$ \\
Thermal softening exponent & $m$ & 1.03 & - \\
Taylor-Quinney coefficient & $\chi$ & 0.9 & - \\
\hline
\end{tabular}

Table B.11: Material properties of C-4340 steel using a Johnson-Cook plastic flow law.

[6] A.-S Bonnet-Lebouvier, A Molinari, and P Lipinski. Analysis of the dynamic propagation of adiabatic shear bands. International Journal of Solids and Structures, 39(16):4249 - 4269, 2002.

[7] T.G. Shawki and R.J. Clifton. Shear band formation in thermal viscoplastic materials. Mechanics of Materials, 8(1):13 - 43, 1989.

[8] F. Armero and J.C. Simo. A priori stability estimates and unconditionally stable product formula algorithms for nonlinear coupled thermoplasticity. International Journal of Plasticity, 9(6):749 - 782, 1993.

[9] J. Lemaitre and J.L. Chaboche. Mechanics of Solid Materials. Cambridge University Press, Cambridge, 2nd edition, 1994.

[10] D. Peirce, C.F. Shih, and A. Needleman. A tangent modulus method for rate dependent solids. Computers and Structures, 18(5):875-887, 1984.

[11] R.K. Abu Al-Rub and G.Z. Voyiadjis. A finite strain plastic-damage model for high velocity impact using combined viscosity and gradient localization 
limiters: Part I - theoretical formulation. International Journal of Damage Mechanics, 15(4):293-334, 2006.

[12] S. Li, W.-K. Liu, D. Qian, P.R. Guduru, and A.J. Rosakis. Dynamic shear band propagation and micro-structure of adiabatic shear band. Computer Methods in Applied Mechanics and Engineering, 191(1-2):73-92, 2001. Micromechanics of Brittle Materials and Stochastic Analysis of Mechanical Systems.

[13] T. Belytschko, H.-Y. Chiang, and E. Plaskacz. High resolution twodimensional shear band computations: imperfections and mesh dependence. Computer Methods in Applied Mechanics and Engineering, 119(1-2):1-15, 1994.

[14] M. Zhou, G. Ravichandran, and A.J. Rosakis. Dynamically propagating shear bands in impact-loaded prenotched plates-II. numerical simulations. Journal of the Mechanics and Physics of Solids, 44(6):1007-1032, 1996.

[15] S. Li and W.K. Liu. Numerical simulations of strain localization in inelastic solids using mesh-free methods. International Journal for Numerical Methods in Engineering, 48(9):1285-1309, 2000.

[16] S. Li, W. Hao, and W.-K. Liu. Mesh-free simulations of shear banding in large deformation. International Journal of Solids and Structures, 37(4850):7185-7206, 2000.

[17] W.K. Liu and C. McVeigh. Predictive multiscale theory for design of heterogeneous materials. Computational Mechanics, 42(2):147-170, 2008.

[18] C. McVeigh and W.K. Liu. Multiresolution continuum modeling of microvoid assisted dynamic adiabatic shear band propagation. Journal of the Mechanics and Physics of Solids, 58(2):187 - 205, 2010.

[19] B. Loret and J.H. Prevost. Dynamic strain localization in elasto-(visco)plastic solids, part 1. general formulation and one-dimensional examples. Computer Methods in Applied Mechanics and Engineering, 83(3):247-273, 1990.

[20] E.C. Aifantis. On the role of gradients in the localization of deformation and fracture. International Journal of Engineering Science, 30(10):1279-1299, 1992. 
[21] H. M. Zbib and E. C. Aifantis. On the gradient-dependent theory of plasticity and shear banding. Acta Mechanica, 92(1):209-225, 1992.

[22] L. Anand, O. Aslan, and S. A. Chester. A large-deformation gradient theory for elastic-plastic materials: Strain softening and regularization of shear bands. International Journal of Plasticity, 30-31:116-143, 2012.

[23] R.C. Batra and C.H. Kim. Effect of thermal conductivity on the initiation, growth and bandwidth of adiabatic shear bands. International Journal of Engineering Science, 29(8):949 - 960, 1991.

[24] L. Malcher, F.M. Andrade Pires, and J.M.A. César de Sá. An assessment of isotropic constitutive models for ductile fracture under high and low stress triaxiality. International Journal of Plasticity, 30-31:81-115, 2012.

[25] A. Needleman. Material rate dependence and mesh sensitivity in localization problems. Computer Methods in Applied Mechanics and Engineering, 67(1):69-85, 1988.

[26] S.N. Medyanik, W.K. Liu, and S. Li. On criteria for dynamic adiabatic shear band propagation. Journal of the Mechanics and Physics of Solids, 55(7):1439 - 1461, 2007.

[27] M. Zhou, A. Needleman, and R.J. Clifton. Finite element simulations of shear localization in plate impact. Journal of the Mechanics and Physics of Solids, 42(3):423 - 458, 1994.

[28] T.W. Wright and R.C. Batra. The initiation and growth of adiabatic shear bands. International Journal of Plasticity, 1(3):205 - 212, 1985.

[29] R.C. Batra. The initiation and growth of, and the interaction among, adiabatic shear bands in simple and dipolar materials. International Journal of Plasticity, 3(1):75 - 89, 1987.

[30] C. McAuliffe and H. Waisman. Mesh insensitive formulation for initiation and growth of shear bands using mixed finite elements. Computational Mechanics, 51(5):807-823, 2013.

[31] C. McAuliffe and H. Waisman. A Pian-Sumihara type element for modeling shear bands at finite deformation. Computational Mechanics, 53(5):925940, 2013. 
[32] L. Berger-Vergiat, C. McAuliffe, and H. Waisman. Isogeometric analysis of shear bands. Computational Mechanics, 54(2):503-521, 2014.

[33] J. Lyness and C. Moler. Numerical differentiation of analytic functions. SIAM Journal on Numerical Analysis, 4(2):202-210, 1967.

[34] W. Squire and G. Trapp. Using complex variables to estimate derivatives of real functions. SIAM Review, 40(1):110-112, 1998.

[35] A. Pérez-Foguet, A. Rodríguez-Ferran, and A. Huerta. Numerical differentiation for local and global tangent operators in computational plasticity. Computer Methods in Applied Mechanics and Engineering, 189(1):277296, 2000.

[36] C. Miehe. Numerical computation of algorithmic (consistent) tangent moduli in large-strain computational inelasticity. Computer Methods in Applied Mechanics and Engineering, 134(3-4):223-240, 1996.

[37] L. Berger-Vergiat, X. Chen, and H. Waisman. Explicit and implicit methods for shear band modeling at high strain rates. International Journal of Fracture, under review, 2016.

[38] Y. Saad and M. Schultz. Gmres: A generalized minimal residual algorithm for solving nonsymmetric linear systems. SIAM Journal on Scientific and Statistical Computing, 7(3):856-869, 1986.

[39] H. van der Vorst. Bi-cgstab: A fast and smoothly converging variant of bi-cg for the solution of nonsymmetric linear systems. SIAM Journal on Scientific and Statistical Computing, 13(2):631-644, 1992.

[40] R.S. Tuminaro and C. Tong. Parallel smoothed aggregation multigrid: Aggregation strategies on massively parallel machines. In Proceedings of the 2000 ACM/IEEE Conference on Supercomputing, Supercomputing '00, Washington, DC, USA, 2000. IEEE Computer Society.

[41] M.W. Gee, C.M. Siefert, J.J. Hu, R.S. Tuminaro, and M.G. Sala. ML 5.0 smoothed aggregation user's guide. Technical Report SAND2006-2649, Sandia National Laboratories, 2006.

[42] I. Ipsen. A note on preconditioning nonsymmetric matrices. SIAM Journal on Scientific Computing, 23(3):1050-1051, 2001. 
[43] V. Howle, R. Kirby, and G. Dillon. Block preconditioners for coupled physics problems. SIAM Journal on Scientific Computing, 35(5):S368S385, 2013.

[44] H. Elman, V.E. Howle, J. Shadid, R. Shuttleworth, and R. Tuminaro. A taxonomy and comparison of parallel block multi-level preconditioners for the incompressible Navier-Stokes equations. Journal of Computational Physics, 227(3): 1790 - 1808, 2008.

[45] E.C. Cyr, J.N. Shadid, and R.S. Tuminaro. Stabilization and scalable block preconditioning for the Navier-Stokes equations. Journal of Computational Physics, 231(2):345 - 363, 2012.

[46] S.C. Eisenstat and H.F. Walker. Choosing the forcing terms in an inexact newton method. SIAM Journal on Scientific Computing, 17(1):16-32, 1996.

[47] B.T. Aagaard, M.G. Knepley, and C.A. Williams. A domain decomposition approach to implementing fault slip in finite-element models of quasi-static and dynamic crustal deformation. Journal of Geophysical Research: Solid Earth, 118(6):3059-3079, 2013.

[48] S. Rhebergen, G. Wells, R. Katz, and A. Wathen. Analysis of block preconditioners for models of coupled magma/mantle dynamics. SIAM Journal on Scientific Computing, 36(4):A1960-A1977, 2014.

[49] E.C. Cyr, J.N. Shadid, R.S. Tuminaro, R.P. Pawlowski, and L. Chacón. A new approximate block factorization preconditioner for two-dimensional incompressible (reduced) resistive mhd. SIAM Journal on Scientific Computing, 35(3):701-730, 2013.

[50] X.-C. Cai and M. Sarkis. A restricted additive schwarz preconditioner for general sparse linear systems. SIAM Journal on Scientific Computing, 21(2):792-797, 1999.

[51] Rahul and S. De. An efficient block preconditioner for jacobian-free globallocal multiscale methods. International Journal for Numerical Methods in Engineering, 87(7):639-663, 2011.

[52] C. Farhat and F.-X. Roux. Method of finite element tearing and interconnecting and its parallel solution algorithm. International Journal for Numerical Methods in Engineering, 32(6):1205-1227, 1991. 
[53] O. Lloberas-Valls, D.J. Rixen, A. Simone, and L.J. Sluys. Domain decomposition techniques for the efficient modeling of brittle heterogeneous materials. Computer Methods in Applied Mechanics and Engineering, 200(1316):1577 - 1590, 2011.

[54] H.A. Schwarz. Über einen Grenzübergang durch alternierendes Verfahren. Vierteljahrsschrift der Naturforschenden Gesellschaft in Zürich, 15:272286, 1870.

[55] X.-C. Cai, W.D. Gropp, D.E. Keyes, R.G. Melvin, and D.P. Young. Parallel newton-krylov-schwarz algorithms for the transonic full potential equation. SIAM Journal on Scientific Computing, 19(1):246-265, 1998.

[56] D.E. Keyes and W.D. Gropp. A comparison of domain decomposition techniques for elliptic partial differential equations and their parallel implementation. SIAM Journal on Scientific and Statistical Computing, 8(2):s166s202, 1987.

[57] V. Gupta, C.A. Duarte, I. Babuška, and U. Banerjee. Stable GFEM (SGFEM): Improved conditioning and accuracy of GFEM/XFEM for threedimensional fracture mechanics. Computer Methods in Applied Mechanics and Engineering, 289:355 - 386, 2015.

[58] Shuhai Zhang and Caglar Oskay. Variational multiscale enrichment method with mixed boundary conditions for elasto-viscoplastic problems. Сотрutational Mechanics, 55(4):771-787, 2015.

[59] L. Berger-Vergiat, H. Waisman, B. Hiriyur, R. Tuminaro, and D. Keyes. Inexact schwarz-algebraic multigrid preconditioners for crack problems modeled by extended finite element methods. International Journal for Numerical Methods in Engineering, 90(3):311-328, 2012.

[60] Haim Waisman and Luc Berger-Vergiat. An adaptive domain decomposition preconditioner for crack propagation problems modeled by xfem. International Journal for Multiscale Computational Engineering, 11(6), 2013.

[61] T.J.R. Hughes, J.A. Cottrell, and Y. Bazilevs. Isogeometric analysis: Cad, finite elements, nurbs, exact geometry and mesh refinement. Computer Methods in Applied Mechanics and Engineering, 194(39-41):4135 - 4195, 2005. 
[62] R. Bouclier, T. Elguedj, and A. Combescure. Locking free isogeometric formulations of curved thick beams. Computer Methods in Applied Mechanics and Engineering, 245-246(0):144 - 162, 2012.

[63] Y. Bazilevs, L. Beirao da Veiga, J.A. Cottrell, T.J.R. Hughes, and G. Sangalli. Isogeometric analysis: approximation, stability and error estimates for hrefined meshes. Mathematical Models and Methods in Applied Sciences, 16(07):1031-1090, 2006.

[64] J. Schur. Über Potenzreihen, die im Innern des Einheitskreises beschränkt sind. Journal für die reine und angewandte Mathematik, 147:205-232, 1917.

[65] BF Smith, P Bjørstad, and W Gropp. Domain decomposition: parallel multilevel methods for elliptic partial differential equations. Cambridge University Press, 1996.

[66] X.-C. Cai, M. Dryja, and M. Sarkis. Restricted additive schwarz preconditioners with harmonic overlap for symmetric positive definite linear systems. SIAM Journal on Numerical Analysis, 41(4):1209-1231, 2003.

[67] R.L. Taylor. FEAP - - A Finite Element Analysis Program, April 2011.

[68] G. Karypis and V. Kumar. A Fast and High Quality Multilevel Scheme for Partitioning Irregular Graphs. SIAM Journal on Scientific Computing, 20(1):359-392, January 1998.

[69] W. Gropp, E. Lusk, and A. Skjellum. Using MPI, Third Edition: Portable Parallel Programming with the Message-Passing Interface. MIT Press, 2014.

[70] S. Balay, J. Brown, K. Buschelman, V. Eijkhout, W. D. Gropp, D. Kaushik, M. G. Knepley, L. Curfman McInnes, B. F. Smith, and H. Zhang. PETSc users manual. Technical Report ANL-95/11 - Revision 3.4, Argonne National Laboratory, 2013.

[71] J. Brown, M.G. Knepley, D.A. May, L.C. McInnes, and B. Smith. Composable linear solvers for multiphysics. In Parallel and Distributed Computing (ISPDC), 2012 11th International Symposium on, pages 55-62, June 2012.

[72] B. Smith, L. Curfman McInnes, E. Constantinescu, M. Adams, S. Balay, J. Brown, M. Knepley, and H. Zhang. PETSc's software strategy for the 
design space of composable extreme-scale solvers. Preprint ANL/MCSP2059-0312, Argonne National Laboratory, 2012. DOE Exascale Research Conference, April 16-18, 2012, Portland, OR.

[73] P.R. Amestoy, I.S. Duff, and J.-Y. L'Excellent. Multifrontal parallel distributed symmetric and unsymmetric solvers. Computer Methods in Applied Mechanics and Engineering, 184(2-4):501 - 520, 2000.

[74] P.R. Amestoy, I.S. Duff, J.-Y. L'Excellent, and J. Koster. A fully asynchronous multifrontal solver using distributed dynamic scheduling. SIAM Journal on Matrix Analysis and Applications, 23(1):15-41, 2001.

[75] P. R. Amestoy, A. Guermouche, J.-Y L'Excellent, and S. Pralet. Hybrid scheduling for the parallel solution of linear systems. Parallel Computing, 32(2):136 - 156, 2006. Parallel Matrix Algorithms and Applications (PMAA'04).

[76] A. George and J.W.H. Liu. A fast implementation of the minimum degree algorithm using quotient graphs. ACM Transactions on Mathematical Software, 6(3):337-358, September 1980.

[77] J.W.H. Liu. Modification of the minimum-degree algorithm by multiple elimination. Transactions on Mathematical Software, 11(2):141-153, June 1985.

[78] A. Henderson. ParaView Guide, A Parallel Visualization Application. Kitware Inc., 2008.

[79] J. D. Hunter. Matplotlib: A 2d graphics environment. Computing In Science \& Engineering, 9(3):90-95, 2007.

[80] C. Geuzaine and J.-F. Remacle. Gmsh: a three-dimensional finite element mesh generator with built-in pre- and post-processing facilities. International Journal for Numerical Methods in Engineering, 79(11):1309-1331, 2009.

[81] J.-F. Remacle, J. Lambrechts, B. Seny, E. Marchandise, A. Johnen, and C. Geuzaine. Blossom-quad: a non-uniform quadrilateral mesh generator using a minimum cost perfect matching algorithm. International Journal for Numerical Methods in Engineering, 89(9):1102-1119, 2012. 
[82] J.-F. Remacle, F. Henrotte, T. Carrier-Baudouin, E. Béchet, E. Marchandise, C. Geuzaine, and T. Mouton. A frontal delaunay quad mesh generator using the L $\infty$ norm. International Journal for Numerical Methods in Engineering, 94(5):494-512, 2013.

[83] G. Karypis and V. Kumar. Parallel Multilevel series k-Way Partitioning Scheme for Irregular Graphs. SIAM Review, 41(2):278-300, January 1999.

[84] J. Litonski. Plastic flow of a tube under adiabatic torsion. Bulletin de l'Académie Polonaise des Sciences - Série des Sciences et Techniques, 25(1):7-14, 1977.

[85] L. Berger-Vergiat, C. McAuliffe, and H. Waisman. Parallel preconditioners for monolithic solution of shear bands. Journal of Computational Physics, $304: 359-379,2016$.

[86] J.-F. Kalthoff. Modes of dynamic shear failure in solids. International Journal of Fracture, 101(1):1-31, 2000.

[87] Miguel Arriaga, Colin McAuliffe, and Haim Waisman. Onset of shear band localization by a local generalized eigenvalue analysis. Computer Methods in Applied Mechanics and Engineering, 289:179 - 208, 2015. 